\title{
HERMITIAN MOMENT SEQUENCES
}

BY

\section{J. S. MAC NERNEY}

1. Introduction. Let $S$ be a complex linear space, $Q$ be an inner product for $S$, and $N$ be the corresponding norm: $N(x)=Q(x, x)^{1 / 2}$ for all $x$ in $S$. Suppose that $S$ is complete with respect to $N$, and let $T$ be the set of all linear functions from $S$ into $S$ which are continuous with respect to $N$. The set of complex numbers is regarded as a subset of $T$ and, for each $A$ in $T, A^{*}$ denotes the adjoint of $A$ with respect to $Q, \operatorname{Re} A$ denotes $\left(A+A^{*}\right) / 2$, and $\operatorname{Im} A$ denotes $\left(A-A^{*}\right) / 2 i$. The notation $H \ll K$ (alternatively, $K \gg H$ ) means that each of $H$ and $K$ is in $T$ and $Q(x, H x) \leqq Q(x, K x)$ for all $x$ in $S$ (implying that each of $H$ and $K$ is Hermitian with respect to $Q$, since $S$ is complex). If $H \gg 0$ then $H^{1 / 2}$ denotes that member $A$ of $T$ such that $A \gg 0$ and $A^{2}=H$. By an infinite sequence we mean a function from the set of all nonnegative integers; by an infinite matrix, a function from the set of all ordered pairs of nonnegative integers.

The statement that $C$ is an Hermitian moment sequence means that $C$ is an infinite sequence with values in $T$ and that there exists a function $\phi$ from the real numbers to $T$, which is nondecreasing in the sense that $\phi(u)$ $\ll \phi(v)$ for $u<v$, such that

$$
C_{n}=\int I^{n} d \phi \quad(n=0,1, \cdots),
$$

where $I$ denotes the identity function on the real line. Let it be noted that we may take $\phi(-\infty)=0$, in which case $C_{0}=\phi(+\infty)$. All limits in $T$ are to be interpreted as "strong" limits with respect to the norm $N$, unless otherwise specified.

The main problem for which we provide new solutions in this paper is twofold: to characterize all Hermitian moment sequences and to find connections between these sequences and certain continued fractions. We find that each of the following is a complete characterization, i.e., is a necessary and sufficient condition that the infinite sequence $C$ with values in $T$ be an Hermitian moment sequence:

(I) $C_{n}^{*}=C_{n}(n=0,1, \cdots)$ and there should exist an analytic function $f$, from the upper half of the complex plane to $T$, such that $\operatorname{Im} f(z) \ll 0$ for $\operatorname{Im} z>0$ and, for each positive integer $n$,

Presented to the Society, November 21, 1959, under the title Operator-moment problems, and on January 23, 1961; received by the editors February 1, 1961. 


$$
h_{n}(z)=z^{n+1} f(z)-\sum_{k=0}^{n-1} z^{n-k} C_{k}
$$

is weakly bounded as $\operatorname{Im} z \rightarrow+\infty$ with $\operatorname{Re} z=0$-in the sense that for each point $x$ in $S$ there exist positive numbers $p$ and $q$ such that if $u>p$ then $\left|Q\left(h_{n}(i u) x, x\right)\right|<q$.

(II) There should exist a member $A_{0}$ of $T$ such that $A_{0} A_{0}^{*}=C_{0}$ and an infinite matrix $M$ with values in $T$ such that

$$
M(i, j)=0 \quad \text { for }|i-j|>1, \quad M(i, j)^{*}=M(j, i) \quad(i, j=0,1,2, \cdots)
$$

and $A_{0} M^{k}(0,0) A_{0}^{*}=C_{k}$ for each positive integer $k$.

(III) There should, for each nonnegative integer $n$, exist a number interval $\left[a_{n}, b_{n}\right]$ such that

$$
a_{n} \sum_{i, j=0}^{n} Q\left(x_{i}, C_{i+j} x_{j}\right) \leqq \sum_{i, j=0}^{n} Q\left(x_{i}, C_{i+j+1} x_{j}\right) \leqq b_{n} \sum_{i, j=0}^{n} Q\left(x_{i}, C_{i+j} x_{j}\right)
$$

for all sequences $\left\{x_{i}\right\}_{0}^{n}$ with values in the space $S$.

The characterization (I) is a direct extension of known results about numerical moment sequences [3], an extension found in 1952 [8] with the added axiom that the inner product space $\{S, Q\}$ is separable. The arguments given in [8] concerning (I) carry over to the present setting with only minor modification, as do arguments supporting the following proposition (and no further proof will be offered). If $f$ is a function from the upper half of the complex plane to $T$ then the following two statements are equivalent:

(a) $f$ is analytic, $\operatorname{Im} f(z) \ll 0$ for $\operatorname{Im} z>0$, and $z f(z)$ is weakly bounded as $\operatorname{Im} z \rightarrow+\infty$ with $\operatorname{Re} z=0$.

(b) There is a nondecreasing function $\phi$ from the real line to $T$ such that each of the limits $\phi(-\infty)$ and $\phi(+\infty)$ exists, and $f$ is the Stieltjes transform of $\phi$, i.e.,

$$
f(z)=\int \frac{1}{z-I} d \phi \quad \text { for } \operatorname{Im} z>0
$$

This latter equivalence is one of the essential tools used here, along with ideas developed in $[9 ; 10]$, to establish (II) and (III).

As well as including solvability criteria for the classical Stieltjes [16] and Hamburger [3] problems (since $T$ "contains" the values of numerical sequences), these results include corresponding criteria for matrix-moment problems as considered by KreIn [7] and by Wall [22]-since the latter correspond to finite dimensional $S$ (see Theorem 4, and Remark 1 following Theorem 3).

Apropos of the Stieltjes moment problem: the infinite sequences $C$ with values in $T$ such that $C_{2 n+1}=0(n=0,1, \cdots)$ which are Hermitian moment 
sequences are easily seen to be those which are generated by a nondecreasing $\phi$ which is odd-in the sense that $\phi(-u)=-\phi(u)$ for all real $u$-and are characterized (Theorem 5 ) by the condition (II) with the proviso $M(i, i)=0$ $(i=0,1, \cdots)$. With $B_{n}=C_{2 n}(n=0,1, \cdots)$, these sequences are also characterized by the condition that $B$ satisfy (III) with the proviso $a_{n} \geqq 0$ $(n=0,1, \cdots)$; indeed, we prove (Theorem 6$)$ that if $I$ is an infinite matrix with values in $T$ then the following two statements are equivalent:

(1) $L(i, j)=0$ for $|i-j|>1(i, j=0,1,2, \cdots)$ and

$$
\sum_{i, j=0}^{n} Q\left(x_{i}, L(i, j) x_{j}\right) \geqq 0
$$

for $n=0,1, \cdots$ and each sequence $\left\{x_{i}\right\}_{0}^{n}$ with values in the space $S$.

(2) There is an infinite matrix $M$ with values in $T$ such that

$$
M(i, j)=0 \quad \text { for }|i-j| \neq 1, \quad M(i, j)^{*}=M(j, i),
$$

and $L(i, j)=M^{2}(2 i, 2 j)(i, j=0,1,2, \cdots)$.

If statement (2) holds then $L^{k}(0,0)=M^{2 k}(0,0)(k=1,2, \cdots)$.

For the case that a single interval $[a, b]$ suffices $(n=0,1, \cdots)$ in the characterization (III), an equivalent formulation is: there should exist a nondecreasing function $\phi$ from $[a, b]$ to $T$ such that

$$
C_{n}=\int_{a}^{b} I^{n} d \phi \quad(n=0,1, \cdots) ;
$$

for such a $\phi$, and each $A_{0}$ in $T$ such that $A_{0} A_{0}^{*}=C_{0}$, we find that there is an infinite matrix $M$ which satisfies (II) and which gives rise to a continued fraction expansion for complex $z$ in the region $\operatorname{Ext}[a, b]$ -

$$
\begin{aligned}
\int_{a}^{b} \frac{1}{z-I} d \phi & =\text { Limil } A_{0} \cdot \frac{A_{0}^{*}}{z-B_{1}-A_{1}} \cdot \frac{A_{1}^{*}}{z-B_{2}-A_{2}} \cdot \frac{A_{2}^{*}}{z-B_{3}-\cdot} . \\
& =\text { Limit } A_{0}\left(z-B_{1}-A_{1}\left(z-B_{2}-\cdots\right)^{-1} A_{1}^{*}\right)^{-1} A_{0}^{*},
\end{aligned}
$$

where $B_{p}=M(p-1, p-1)$ and $A_{p}=M(p-1, p)(p=1,2, \cdots)$ and convergence is uniform in $z$ over each closed bounded subset of Ext $[a, b]$. This case is also characterized by an extension (Lemma 8 ) of the representation theorem due to F. Riesz [12] for "positive linear functionals" on the space of continuous functions from $[a, b]$ to the numbers (see, also, Christian [2] concerning a further extension). The subcase, in which $[a, b]=[-1,1]$ and $\phi$ is odd, is characterized by the following special form for the preceding continued fraction expansion (an extension of H. S. Wall's characterization [19] 
of totally monotone numerical sequences):

$$
B_{p}=0 \text { and } A_{p}=\left[1-m_{p-1}\right]^{1 / 2} u_{p} m_{p}^{1 / 2} \quad(p=1,2, \cdots),
$$

where $m_{0}=0$, and $0 \ll m_{p} \ll 1$, and $u_{p}^{*} u_{p}$ is the projection of $S$ onto the closure of $m_{p}(S)$ with respect to $N$.

Finally, we find that there is a notion of "bounded variation" for functions from the real line to $T$ which provides - essentially as corollaries to the preceding analysis-an analogue (Theorem 9) of the characterization of moment sequences of numerical functions of bounded variation on the unit-interval [5] as differences of totally monotone numerical sequences, and an extension (Theorem 10) to the present setting of the Boas-Pólya result [1] that every infinite numerical sequence is the moment sequence of a numerical function of bounded variation which is constant on the set of negative numbers.

2. Definitions and basic lemmas. In addition to the notational conventions mentioned in the introduction, if $A$ belongs to $T$ then $A^{-1}$ denotes the inverse of the contraction of $A$ to the closure of $A^{*}(S)$. We use $\bar{N}$ to denote the norm for $T$ defined by:

$$
\bar{N}(A)=\text { L.U.B. } N(A x) \quad \text { for } N(x)=1 .
$$

If $A$ is in $T$ then $|A|$ denotes the transformation $\left[A A^{*}\right]^{1 / 2}$; this is consistent with standard notation (e.g., $[15 \mathrm{a}$, p. 277]) in case $A$ is Hermitian with respect to $Q$. If $H \gg 0, H^{-1 / 2}$ denotes $\left(H^{1 / 2}\right)^{-1}$.

Lemma 1. If $D$ is in Then $|D|(S)=D(S)$ and is the subset of $S$ to which $z$ belongs only in case there is a nonnegative number $m$ such that $|Q(z, x)|$ $\leqq m N\left(D^{*} x\right)$ for all $x$ in $S$, in which case $N\left(D^{-1} z\right)$ is the least such number $m$.

For a proof, see Theorem 1 of [9] or Lemma 3.1 of [10].

LemMa 2. Supposing that each of $D$ and $E$ is in $T, D D^{*}=E E^{*}, U=D^{-1} E$, and $V=E^{-1} D$, the following are true:

(1) Each of $U$ and $V$ is in $T$, and $U^{*}=V$.

(2) $U^{*} U$ is the projection of $S$ onto the closure of $E^{*}(S)$.

(3) $V^{*} V$ is the projection of $S$ onto the closure of $D^{*}(S)$.

(4) For each $x$ and $y$ in $D(S), Q\left(D^{-1} x, D^{-1} y\right)=Q\left(E^{-1} x, E^{-1} y\right)$.

REMARK 1. This lemma is a refinement of the result [15a, p. 286] that every member of $T$ is the composite of a nonnegative Hermitian transformation with a partially isometric transformation (let $E=|D|$ ).

REMARK 2. It follows from Lemma 2 that, if each of $A, B$, and $D$ is in $T$, each of $A(S)$ and $B(S)$ lies in $D(S)$, and each of $D^{-1} A$ and $D^{-1} B$ is also in $T$, then the transformation $\left\{|D|^{-1} A\right\} *\left\{|D|^{-1} B\right\}$, which we have previously indicated by $A^{*}\left[D D^{*}\right]^{-1} B$ (see $[9$, Theorem 4$]$ ), is the transformation $\left\{D^{-1} A\right\} *\left\{D^{-1} B\right\}$. 
Proof. That each of $U$ and $V$ is in $T$ follows from Lemma 1, with the observation that, for $x$ and $y$ in $S, N\left(D^{*} x\right)=N\left(E^{*} x\right)$ so that

$$
|Q(E y, x)| \leqq N(y) N\left(D^{*} x\right)
$$

and

$$
|Q(D y, x)| \leqq N(y) N\left(E^{*} x\right),
$$

whence $N(U y) \leqq N(y)$ and $N(V y) \leqq N(y)$. Let $P_{1}$ and $P_{2}$ be projections (with respect to $Q$ ) of $S$ onto the closures of $D^{*}(S)$ and $E^{*}(S)$, respectively. Now, $U E^{*}=D^{*}=(E V)^{*}=V^{*} E^{*}$, so that by continuity we have $\left(U P_{2}\right)^{*}=P_{2} V$. Since $U\left[1-P_{2}\right]=\left[1-P_{2}\right] V=0$, then $U^{*}=V$. Since $U^{*} U E^{*}=U^{*} D^{*}=(D U)^{*}$ $=E^{*}$ then, again by continuity, we see that $U^{*} U=U^{*} U P_{2}=P_{2}$. Similarly $V^{*} V=P_{1}$. The last assertion of the lemma follows from the observation that for each $x$ and $y$ in $S$

$$
Q\left(P_{1} x, P_{1} y\right)=Q\left(P_{1} x, V^{*} V P_{1} y\right)=Q\left(V P_{1} x, V P_{1} y\right),
$$

and this completes our proof.

Lemma 3. Suppose that each of $A, B$, and $H$ is in $T, P_{1}$ is the projection of $S$ onto the closure of $A^{*}(S), P_{2}$ is the projection of $S$ onto the closure of $B^{*}(S)$, and there is a number $m$ such that

$$
|Q(x, H y)| \leqq m N\left(A^{*} x\right) N\left(B^{*} y\right) \quad \text { for each } x \text { and } y \text { in } S .
$$

Then $\left(B^{-1}\left[A^{-1} H\right]^{*}\right)^{*}=A^{-1}\left[B^{-1} H^{*}\right]^{*}$ and is the only member $G$ of $T$ such that $H=A G B^{*}$ and $P_{1} G P_{2}=G$.

REMARK. If $H^{*}=H$ and $B=A$ then $G^{*}=G$; moreover, one sees that if $[a, b]$ is a number interval then the condition that

$$
a A A^{*} \ll B \ll b A A^{*}
$$

implies that $a P_{1} \ll G \ll b P_{1}$, and if $H=A C^{*}$ then $G A^{*}=P_{1} C^{*}$.

Proof. Successive applications of Lemma 1 show that, for each $x$ and $y$ in $S$,

$$
N\left(A^{-1} H y\right) \leqq m N\left(B^{*} y\right)
$$

and

$$
N\left(B^{-1}\left[A^{-1} H\right]^{*} x\right) \leqq m N(x) .
$$

Let $G=\left(B^{-1}\left[A^{-1} H\right]^{*}\right)^{*}$. Then $B G^{*}=\left[A^{-1} H\right]^{*}$ so that $H=A G B^{*}$. Now, since $G^{*}=B^{-1}\left[A^{-1} H\right]^{*}$, it follows that $P_{2} G^{*}=G^{*}$ so that $G P_{2}=G$. Since $G B^{*}$ $=A^{-1} H$, it follows that $P_{1} G B^{*}=G B^{*}$ so that $P_{1} G P_{2}=G P_{2}$. If $F$ is a member of $T$ such that $H=A F B^{*}$ and $P_{1} F P_{2}=F$, then we see, successively, that:

$A G B^{*}=A F B^{*}, A G P_{2}=A F P_{2}, A G=A F, G^{*} P_{1}=F^{*} P_{1}, G=P_{1} G=P_{1} F=F$. 
Now, two more applications of Lemma 1 show that for each $x$ and $y$ in $S$

$$
N\left(B^{-1} H^{*} x\right) \leqq m N\left(A^{*} x\right)
$$

and

$$
N\left(A^{-1}\left[B^{-1} H^{*}\right] * y\right) \leqq m N(y) .
$$

Let $F=A^{-1}\left[B^{-1} H^{*}\right]^{*}$. Then $P_{1} F=F$ and $F^{*} A^{*}=B^{-1} H^{*}$. Hence $H=A F B^{*}$ and $P_{2} F^{*} A^{*}=F^{*} A^{*}$. Thus we see that $P_{2} F^{*} P_{1}=F^{*} P_{1}$ and $P_{1} F P_{2}=P_{1} F$. This completes our proof.

Lemma 4. If $A$ is in $T$ and $b$ is a positive number and $\operatorname{Re} A \gg b$ then $A$ is reversibly continuous from $S$ onto $S$ and $\bar{N}\left(A^{-1}\right) \leqq 1 / b$.

Indication of proof. From the hypothesis, for each $x$ in $S$,

$$
2 b N(x)^{2}=2 b Q(x, x) \leqq Q(A x, x)+Q(x, A x) \leqq 2 N(x) N(A x),
$$

so that $b N(x) \leqq N(A x)$, and similarly $b N(x) \leqq N\left(A^{*} x\right)$; if $z$ is in $S$,

$$
|Q(z, x)| \leqq\{N(z) / b\} N(A x)
$$

and

$$
|Q(z, x)| \leqq\{N(z) / b\} N\left(A^{*} x\right) .
$$

By Lemma $1, A^{*}(S)=A(S)=S$, etc.

Lemma 5. Suppose $\phi$ is a nondecreasing function from the real line into $T$ with $\phi(-\infty)=0$ and $\phi(+\infty)=1, f$ is the Stieltjes transform of $\phi$, and the following integrals exist:

$$
C_{1}=\int I d \phi \quad \text { and } \quad C_{2}=\int I^{2} d \phi
$$

There exists a nondecreasing function $\theta$ from the real line into $T$, with $\theta(-\infty)=0$ and $\theta(+\infty)=C_{2}-C_{1}^{2}$, such that if $g$ is the Stieltjes transform of $\theta$ then

(1) for $\operatorname{Im} z>0, f(z)$ is reversibly continuous from $S$ onto $S$ and

$$
f(z)^{-1}=z-C_{1}-g(z),
$$

(2) if all the integrals $C_{n}=\int I^{n} d \phi(n=3,4, \cdots)$ exist then for each positive integer $n$

$$
\int I^{n} d \theta=C_{n+2}-C_{n+1} C_{1}-\sum_{k=0}^{n-1} C_{n-k} \int I^{k} d \theta
$$

REMARK 1. As noted in the introduction, an analytic function $g$ from the upper half of the complex plane into $T$ is the Stieltjes transform of a nondecreasing function $\theta$ from the real line into $T$, such that $\theta(-\infty)=0$ and $\theta(+\infty)$ exists, only in case $\operatorname{Im} g(z) \ll 0$ for $\operatorname{Im} z>0$ and $z g(z)$ is weakly bounded 
as $\operatorname{Im} z \rightarrow+\infty$ with $\operatorname{Re} z=0$; in this case [8, Theorem 3.2 and Remarks], $z g(z)$ has weak limit $\theta(+\infty)$.

REMARK 2. In this Lemma, the integrals $C_{1}$ and $C_{2}$ exist only in case [8, Theorem 3.3] there exists an Hermitian member $H$ of $T$ such that $z^{3} f(z)-z^{2}$ $-z H$ is weakly bounded as $\operatorname{Im} z \rightarrow+\infty$ with $\operatorname{Re} z=0$, in which case $H=C_{1}$ and $z^{3} f(z)-z^{2}-z H$ has weak limit $C_{2}$.

REMARK 3. It follows from part (1) of this Lemma that if $C_{2}=C_{1}^{2}$ then $f$ is the resolvent of $C_{1}$ and $\phi$ is essentially the spectral resolution of $C_{1}$. Part (1) we have previously obtained $[8$, Theorem 3.4$]$ for the case that $\{S, Q\}$ is separable; we present an independent argument for the present setting.

Proof. With the suppositions of the Lemma, we show first that, for $\operatorname{Im} z>0, f(z)$ is reversibly continuous from $S$ onto $S$ : we have

$$
\operatorname{Im} f(z)=-(\operatorname{Im} z) \int|z-I|^{-2} d \phi
$$

so that, for each $x$ in the space $S$,

$$
\begin{aligned}
Q(x, x)^{2} & =\left\{\int 1 Q([d \phi] x, x)\right\}^{2} \\
& \leqq\left\{\int|z-I|^{-2} Q([d \phi] x, x)\right\}\left\{\int|z-I|^{2} Q([d \phi] x, x)\right\} \\
& =\{-Q([\operatorname{Im} f(z)] x, x) /(\operatorname{Im} z)\}\left\{Q\left(\left[|z|^{2}-2(\operatorname{Re} z) C_{1}+C_{2}\right] x, x\right)\right\},
\end{aligned}
$$

whence we see that

$$
-\operatorname{Im} f(z) \gg(\operatorname{Im} z) / \bar{N}\left(|z|^{2}-2(\operatorname{Re} z) C_{1}+C_{2}\right) ;
$$

the conclusion follows from Lemma 4 , and we have also

$$
\bar{N}\left(f(z)^{-1}\right) \leqq \bar{N}\left(|z|^{2}-2(\operatorname{Re} z) C_{1}+C_{2}\right) /(\operatorname{Im} z) .
$$

Now, letting the function $g$ be defined by

$$
g(z)=z-C_{1}-f(z)^{-1} \quad \text { for } \operatorname{Im} z>0,
$$

and using the preceding estimate for $\bar{N}\left(f(z)^{-1}\right)$, it is easy to see that $g$ is analytic-indeed that $g^{\prime}(z)=1+f(z)^{-1}\left[f^{\prime}(z)\right] f(z)^{-1}$. By judicious use of Schwarz's inequality as before, e.g.,

$$
\begin{aligned}
|Q([z f(z)-1] x, y)| & =\left|\int \frac{I}{z-I} Q([d \phi] x, y)\right| \\
& \leqq \frac{1}{\operatorname{Im} z}\{Q(x, x)\}^{1 / 2}\left\{Q\left(C_{2} y, y\right)\right\}^{1 / 2},
\end{aligned}
$$

we obtain the following estimates, for $\operatorname{Im} z>0$ and $\operatorname{Re} z=0$ : 


$$
\begin{aligned}
\bar{N}\left([z f(z)]^{-1}\right) & \leqq \bar{N}\left(1+C_{2} /(\operatorname{Im} z)^{2}\right), \\
\bar{N}\left(z^{2} f(z)-z\right) & \leqq \bar{N}\left(C_{2}\right)^{1 / 2}, \\
\bar{N}\left(z^{3} f(z)-z^{2}-z C_{1}\right) & \leqq \bar{N}\left(C_{2}\right) .
\end{aligned}
$$

Hence, by the identity

$$
z g(z)=[z f(z)]^{-1}\left\{z^{3} f(z)-z^{2}-z C_{1}-\left[z^{2} f(z)-z\right] C_{1}\right\},
$$

we have the following estimate, for $\operatorname{Im} z>0$ and $\operatorname{Re} z=0$ :

$$
\bar{N}(z g(z)) \leqq \bar{N}\left(1+C_{2} /(\operatorname{Im} z)^{2}\right)\left\{\bar{N}\left(C_{2}\right)+\bar{N}\left(C_{2}\right)^{1 / 2} \bar{N}\left(C_{1}\right)\right\} .
$$

Therefore (see Remarks 1 and 2 preceding this proof), there is a nondecreasing function $\theta$ from the real line into $T$, such that $\theta(-\infty)=0$ and $\theta(+\infty)$ exists, of which $g$ is the Stieltjes transform; now, since

$$
z g(z)=\{1-z f(z)\}[z g(z)]+z \int \frac{I^{2}}{z-I} d \phi-\left\{z^{2} f(z)-z\right\} C_{1},
$$

which has weak limit $C_{2}-C_{1}^{2}$ as $\operatorname{Im} z \rightarrow+\infty$ with $\operatorname{Re} z=0$, it follows that $C_{2}-C_{1}^{2}=\theta(+\infty)$. This establishes part (1).

Concerning (2) now, let $B$ be the infinite sequence defined by

$$
B_{0}=C_{2}-C_{1}^{2} \text { and } B_{n}=C_{n+2}-C_{n+1} C_{1}-\sum_{k=0}^{n-1} C_{n-k} B_{k} \quad(n=1,2, \cdots) .
$$

Inductively we see that $B_{n}^{*}=B_{n}(n=0,1, \cdots)$. For $\operatorname{Im} z>0$, we let

$$
h_{0}(z)=z g(z) \quad \text { and } \quad h_{n+1}(z)=z\left\{h_{n}(z)-B_{n}\right\} \quad(n=0,1, \cdots) .
$$

We show, inductively, that $\bar{N}\left(h_{n}(z)\right)$ is bounded for $\operatorname{Im} z>0$ with $\operatorname{Re} z$ $=0(n=1,2, \cdots)$, and this will establish (2) and complete our proof of the lemma (see Remark 2 preceding this proof). From the last representation for $z g(z)$ in the preceding paragraph, we find

$$
h_{1}(z)=\int \frac{z I^{3}}{z-I} d \phi-\left\{\int \frac{z I^{2}}{z-I} d \phi\right\} C_{1}-\left\{\int \frac{z I}{z-I} d \phi\right\} h_{0}(z) .
$$

From this it is easy to see inductively that, for $n=2,3, \cdots$,

$$
\begin{aligned}
h_{n}(z)= & \int \frac{z I^{n+2}}{z-I} d \phi-\left\{\int \frac{z I^{n+1}}{z-I} d \phi\right\} C_{1} \\
& -\sum_{k=0}^{n-2}\left\{\int \frac{z I^{n-k}}{z-I} d \phi\right\} B_{k}-\left\{\int \frac{z I}{z-I} d \phi\right\} h_{n-1}(z) .
\end{aligned}
$$

By using Schwarz's inequality as in the preceding paragraph, we find that for $\operatorname{Im} z>0$ with $\operatorname{Re} z=0$ and each positive integer $n\left(C_{0}=1\right)$ 


$$
\bar{N}\left(\int \frac{z I^{2 n-1}}{z-I} d \phi\right) \leqq \bar{N}\left(C_{2 n-2}\right)^{1 / 2} \bar{N}\left(C_{2 n}\right)^{1 / 2}
$$

and

$$
\bar{N}\left(\int \frac{z I^{2 n}}{z-I} d \phi\right) \leqq \bar{N}\left(C_{2 n}\right) .
$$

This provides the desired bounds on $\bar{N}\left(h_{n}(z)\right)$ and completes our proof.

LEMмA 6. If $\theta$ is a nondecreasing function from the real line into $T$ and $\theta(-\infty)=0$ and $A$ is a member of $T$ such that $A A^{*}=\theta(+\infty)$, then the formula

$$
\theta_{1}(u)=A^{-1}\left[A^{-1} \theta(u)\right]^{*}
$$

defines a nondecreasing function $\theta_{1}$ from the real line into $T$ such that $\theta_{1}(-\infty)=0$ and $\theta_{1}(+\infty)$ is the projection of $S$ onto the closure of $A^{*}(S)$; if, moreover, $P$ is that projection then the formulas

$$
\phi_{1}(u)=\theta_{1}(u) \quad \text { if } u<0, \quad \phi_{1}(u)=\theta_{1}(u)+1-P \quad \text { if } u \geqq 0
$$

define a nondecreasing function $\phi_{1}$ from the real line into $T$, with Stieltjes transform $f_{1}$ and $\phi_{1}(-\infty)=0$ and $\phi_{1}(+\infty)=1$, such that

$$
A f_{1}(z) A^{*}=\int \frac{1}{z-I} d \theta \quad \text { for } \operatorname{Im} z>0
$$

and also

$$
A\left\{\int I^{n} d \phi_{1}\right\} A^{*}=\int I^{n} d \theta \quad(n=0,1, \cdots)
$$

provided only that all the latter integrals exist.

Indication of proof. That $\theta_{1}$ makes sense and is nondecreasing, follows from Lemma 3 and the Remark immediately thereafter. By Lemma 2, the projection $P$ (of $S$ onto $A^{*}(S)$ ) is given by the formula

$$
P=\left(A^{-1}|A|\right)\left(A^{-1}|A|\right)^{*}=A^{-1}\left(A^{-1}\left[A A^{*}\right]\right)^{*} .
$$

All other assertions of the lemma follow from the following type of consideration (based on Lemma 3 ): if $H$ is an infinite sequence with values in $T$ such that $0 \ll H_{n} \ll H_{n+1} \ll A A^{*}(n=0,1, \cdots)$, and $K$ is the limit of $H$, then

(a) $0 \ll A^{-1}\left(A^{-1} H_{n}\right) * \ll A^{-1}\left(A^{-1} H_{n+1}\right) * \ll 1(n=0,1, \cdots)$,

(b) $A^{-1}\left(A^{-1} H_{n}\right)^{*} \rightarrow J$ such that $P J P=J=J^{*}$, and

(c) $A J A^{*}=K$, so that $J=A^{-1}\left(A^{-1} K\right)^{*}$.

Lemma 7. Suppose $S_{0}$ is a linear subset of $S$ which is dense in $S$, each of $A$ and $B$ is a linear function from $S_{0}$ into $S$, 


$$
Q(A x, y)=Q(x, B y) \quad \text { and } \quad \operatorname{Im} Q(A x, x) \leqq 0
$$

for all $x$ and $y$ in $S_{0}, \bar{A}$ denotes the closure of $A$ (as a subset of $S \times S$ ), and $\bar{B}$ denotes the closure of $B$. If $S_{1}$ is the initial set (i.e., "domain") of $\bar{A}$ and $S_{2}$ is the initial set of $\bar{B}$, there exists a nondecreasing function $\phi$ from the real line into $T$, with Stieltjes transform $f$ and $\phi(-\infty)=0$ and $\phi(+\infty)=1$, such that for $\operatorname{Im} z>0$

(1) $f(z)\{z-\bar{A}\} x=x$ for each $x$ in $S_{1}$, and

(2) $f(z)^{*}\left\{z^{*}-\bar{B}\right\} y=y$ for each $y$ in $S_{2}$.

REMARK. We have earlier [8, Theorem 2.6 and Corollary 3.2(a)] given essentially this result for separable spaces, couched in the language of infinite complex matrices. The case that $S$ is finite dimensional, we set aside as completely taken care of by our results with matrices [8]. Ideas occurring in the following twofold proof have been considered in somewhat more detail by von Neumann in [18], especially pp. 98-103 for the first argument, and pp. 82-92 for the second.

Proof for separable $\{S, Q\}$. Let $s$ be an infinite sequence with values in $S_{0}$ such that the following three conditions hold:

(i) the set $\left[\left\{s_{0}, A s_{0}\right\},\left\{s_{1}, A s_{1}\right\}, \cdots\right]$ is dense in $\bar{A}$,

(ii) the set $\left[\left\{s_{0}, B s_{0}\right\},\left\{s_{1}, B s_{1}\right\}, \cdots\right]$ is dense in $\bar{B}$, and

(iii) the set $\left[s_{0}, s_{1}, s_{2}, \cdots\right]$ is dense in $S$ itself.

Let $\left[t_{0}, t_{1}, t_{2}, \cdots\right]$ be a maximal orthonormal set constructed from $s$ by the usual variation of the Hilbert-Schmidt process [18, Theorem 12.18]. Let $C$ be the infinite complex matrix defined by:

$$
C_{i j}=Q\left(A t_{j}, t_{i}\right)=Q\left(t_{j}, B t_{i}\right) .
$$

Now $\sum_{k}\left|C_{k j}\right|^{2}=N\left(A t_{j}\right)^{2}$ and $\sum_{k}\left|C_{i k}\right|^{2}=N\left(B t_{i}\right)^{2}(i, j=0,1,2, \cdots)$, and for each positive integer $n$ and each complex number sequence $\left\{z_{i}\right\}_{0}^{n}$

$$
\operatorname{Im} \sum_{i, j=0}^{n} z_{i}^{*} C_{i j} z_{j}=\operatorname{Im} Q\left(A\left[\sum_{j=0}^{n} z_{j} t_{j}\right], \quad \sum_{i=0}^{n} z_{i} t_{i}\right) \leqq 0 .
$$

Hence [8, Theorem 2.6 and Corollary 3.2(a)], there exists a nondecreasing function $\phi$ from the real line into $T$, with Stieltjes transform $f$ and $\phi(-\infty)=0$ and $\phi(+\infty)=1$, such that for $\operatorname{Im} z>0$ the matrix $D(z)$ defined by

$$
D(\mathrm{z})_{i j}=Q\left(f(z) t_{j}, t_{i}\right)
$$

is a reciprocal of $\{z \delta-C\}$ (where $\delta$ is the "identity" matrix); thus we have, for $i, j=0,1,2, \cdots$,

$$
Q\left(t_{j}, t_{i}\right)=\sum_{k} D(z)_{i k}\left\{z \delta_{k j}-C_{k j}\right\}=Q\left(\{z-A\} t_{j}, f(z)^{*} t_{i}\right)
$$

which implies (1), and

$$
Q\left(t_{j}, t_{i}\right)=\sum_{k}\left\{z \delta_{i k}-C_{i k}\right\} D(z)_{k j}=Q\left(f(z) t_{j},\left\{z^{*}-B\right\} t_{i}\right)
$$


which implies (2). These latter implications are direct consequences of our requirements (i) and (ii), respectively, on the original sequence $s$.

Proof for nonseparable $\{S, Q\}$. There exists [18, Theorem 14.10] a collection $G$ of mutually orthogonal, separable, closed linear subspaces of $S$ such that

(i) each member of $G$ reduces each of $\bar{A}$ and $\bar{B}$, and

(ii) the members of $G$ span all of $S$.

Supposing $M$ is a member of $G, P_{M}$ is the projection of $S$ onto $M$, and $T_{M}$ is the set of all continuous linear functions from $M$ into $M$, we deduce the following facts (aided by [18, Theorem 14.13]):

(a) $P_{M}\left(S_{0}\right)$ is dense in $M$ and lies in the intersection $S_{M}$ of $P_{M}\left(S_{1}\right)$ with $P_{M}\left(S_{2}\right)$.

(b) The contraction $A_{M}$ of $\bar{A}$ to $S_{M}$ has closure $\bar{A}_{M}$ equal to the contraction of $\bar{A}$ to $P_{M}\left(S_{1}\right)$, and likewise for $B_{M}$ and $P_{M}\left(S_{2}\right)$.

(c) For each $x$ and $y$ in $S_{M}$

$$
Q\left(A_{M} x, y\right)=Q\left(x, B_{M} y\right) \quad \text { and } \quad \operatorname{Im} Q\left(A_{M} x, x\right) \leqq 0 .
$$

(d) There is a nondecreasing function $\phi_{M}$ from the real line into $T_{M}$, with Stieltjes transform $f_{M}$ and $\phi_{M}(-\infty)=0$ and $\phi_{M}(+\infty)=1$ in $T_{M}$, such that for $\operatorname{Im} z>0$

(1 $\left.1_{M}\right) f_{M}(z)\left\{z-\bar{A}_{M}\right\} x=x$ for each $x$ in $P_{M}\left(S_{1}\right)$, and

(2 $\left.2_{M}\right) f_{M}(z)^{*}\left\{z^{*}-\bar{B}_{M}\right\} y=y$ for each $y$ in $P_{M}\left(S_{2}\right)$.

The proof is readily completed now by appropriate "summation" over the members of $G$ [18, Theorem 14.12], and Lemma 7 is established.

Lemma 8. Suppose $[a, b]$ is a number interval, $\mathfrak{e}[a, b]$ is the set of all continuous functions from $[a, b]$ to the real numbers, $E$ is a linear subcollection of $\mathfrak{e}[a, b]$ which is dense in $\mathfrak{C}[a, b]$ in the sense of uniform convergence, and $L$ is $a$ real-linear function from $E$ to $T$. The following two statements are equivalent:

(1) $L(f) \ll L(g)$ for $f \leqq g$ in $E$ (i.e., $f(u) \leqq g(u)$ for $a \leqq u \leqq b)$.

(2) There is a nondecreasing function $\phi$ from $[a, b]$ to $T$ such that

$$
L(f)=\int_{a}^{b} f d \phi \quad \text { for each } f \text { in } E .
$$

REMARK 1. This idea goes back to the work of F. Riesz [12] for the case that $L$ is numerical valued. An extension process, through nondecreasing sequences with values in $E$, which produces the values of $\phi$ as values of an extension of $L$ to characteristic functions of subintervals of $[a, b]$, is due to Riesz [13, Chapter V; 14]. The present lemma is essentially proved in [15, Chapter VII] in connection with Hilbert's spectral resolution theorem: there we have a member $H$ of $T$ such that $a \ll H \ll b, E$ is the class of real polynomials on $[a, b]$, and $L(f)=f(H)$ for all $f$ in $E$-so that $L$ has the additional property of being multiplicative in the sense that $L(f \cdot g)=L(f) L(g)$ for $f$ and $g$ in $E$ (which is equivalent to there being a $\phi$ which is projection valued). 
REMARK 2. An extension to a more general setting has been indicated by R. R. Christian $[2, \S I I I]: E$ is replaced by the bounded real continuous functions on a normal topological space, and $T$ by a Dedekind complete partially ordered vector space.

Indication of proof. Clearly (2) implies (1). Supposing (1) to be true, one carries out the following procedure.

(i) Extend $L$ to the set $E^{\prime}$ of all upper semicontinuous functions on $[a, b]$ which are bounded below, the extension $L^{\prime}$ being additive and homogeneous with respect to positive scalars and having property (1) on $E^{\prime}$ : if $f$ is in $E^{\prime}$ and $F$ is a nonincreasing sequence, with values in $E$ and limit $f$ on $[a, b]$, then $L^{\prime}(f)$ is the limit of $L(F)$.

(ii) Extend $L^{\prime}$ linearly to the set $E^{\prime \prime}$ of all differences of functions in $E^{\prime}$, the extension $L^{\prime \prime}$ being real-linear and having property (1) on $E^{\prime \prime}$ : if $f$ is in $E^{\prime \prime}$ and $f=g_{1}-g_{2}$, with $g_{1}$ and $g_{2}$ in $E^{\prime}$, then $L^{\prime \prime}(f)=L^{\prime}\left(g_{1}\right)-L^{\prime}\left(g_{2}\right)$.

(iii) Define $\phi(a)$ to be 0 and, for $u$ in $(a, b], \phi(u)$ to be $L^{\prime}\left(1_{[a, u]}\right)$ where $1_{[a, u]}(t)$ is 1 or 0 accordingly as $t$ is in $[a, u]$ or not: the function $\phi$ is nondecreasing on $[a, b]$ and, if $f$ is in $E$ and $\left\{u_{i}\right\}_{0}^{n}$ is an increasing numerical sequence with $u_{0}=a$ and $u_{n}=b$, and $m_{i}$ and $M_{i}$ denote respectively the minimum and maximum values of $f$ on the interval $\left[u_{i-1}, u_{i}\right](i=1, \cdots, n)$, then

$$
\begin{aligned}
\sum_{1}^{n} m_{i}\left\{\phi\left(u_{i}\right)-\phi\left(u_{i-1}\right)\right\} & =L^{\prime \prime}\left(m_{1} \cdot 1_{\left[a, u_{1}\right]}+\sum_{2}^{n} m_{i} \cdot 1_{\left(u_{i-1}, u_{i}\right]}\right) \\
& \ll L(f) \ll L^{\prime \prime}\left(M_{1} \cdot 1_{\left[a, u_{1}\right]}+\sum_{2}^{n} M_{i} \cdot 1_{\left[u_{i-1}, u_{i}\right]}\right) \\
& =\sum_{1}^{n} M_{i}\left\{\phi\left(u_{i}\right)-\phi\left(u_{i-1}\right)\right\}
\end{aligned}
$$

-which is enough to establish (2).

LemMa 9. If $C$ is an infinite sequence with values in $T$ and $k$ is a positive integer then

$$
\sum_{p=1}^{n}(p / n)^{k}\left(\begin{array}{l}
n \\
p
\end{array}\right) \Delta^{n-p} C_{p} \rightarrow C_{k} \quad \text { as } n \rightarrow \infty
$$

where

$$
\Delta^{m} C_{i}=\sum_{0}^{m}(-1)^{j}\left(\begin{array}{c}
m \\
j
\end{array}\right) C_{i+j} \quad(i, m=0,1,2, \cdots) .
$$

REMARK 1. This lemma was discovered by the author in 1949 , presented in seminar at the University of Texas, but not published. It was rediscovered and published in 1958 by Jakimovski [6] for numerical sequences (see Wells' 1959 paper [23, p. 635] for comments and applications). 
REMARK 2. The lemma remains true if $T$ is replaced by any linear topological space-as a consequence of the following observation about Bernstein polynomials: for $n \geqq k(k, n=1,2,3, \cdots)$

$$
\sum_{p=1}^{n} p^{k}\left(\begin{array}{l}
n \\
p
\end{array}\right)[1-I]^{n-p} I^{p}=\sum_{q=1}^{k}\left(\begin{array}{l}
n \\
q
\end{array}\right) d_{k, q} I^{q},
$$

where $d_{k, 1}=1, d_{k, k}=k$ !, and $d_{k+1, j}=j\left\{d_{k, j-1}+d_{k, j}\right\} \quad(1<j<k+1)$. (See McShane $[11$, p. 119] for a different formulation.)

Definition. For each positive integer $n, S^{n}$ denotes the set of all sequences $\left\{x_{i}\right\}_{0}^{n}$ with values in $S$, and $Q_{n}$ denotes the inner product for $S^{n}$ defined by

$$
Q_{n}(x, y)=\sum_{i=0}^{n} Q\left(x_{i}, y_{i}\right)
$$

we identify the class of linear functions from $S^{n}$ into $S^{n}$, which are continuous with respect to the norm corresponding to $Q_{n}$, with the class $T^{n}$ of functions from the ordered pairs of integers in $[0, n]$ to $T$-in such a way that if $A$ is in $T^{n}$ then

$$
A x=\left\{\sum_{j=0}^{n} A(i, j) x_{j}\right\}_{0}^{n} \quad \text { for } x=\left\{x_{i}\right\}_{0}^{n}
$$

and $A^{*}(i, j)=A(j, i)^{*}(i, j=0,1,2, \cdots)$.

Lemma 10. Suppose $n$ is a positive integer, each of $A$ and $B$ is in $T^{n},\left\{D_{i}\right\}_{0}^{n}$ is a sequence with values in $T$ such that

$$
A^{*} x=\left\{\sum_{j=i}^{n} D_{i}^{-1} B(i, j) x_{j}\right\}_{0}^{n} \quad \text { for } x \text { in } S^{n},
$$

where $B(i, i)=D_{i} D_{i}^{*}$ and $B(i, j)(S)$ is a subset of $D_{i}(S)$ for $i \leqq j$, and $p_{i}$ is the projection of $S$ onto the closure of $D_{i}^{*}(S)(i=0, \cdots, n)$. In order that the member $y$ of $S^{n}$ should belong to the closure of $A^{*}\left(S^{n}\right)$, it is necessary and sufficient that $p_{i} y_{i}=y_{i}$ for $i=0, \cdots, n$.

REMARK. We now see that-with the suppositions of Lemma 10-if $P$ is the projection of $S^{n}$ onto the closure of $A^{*}\left(S^{n}\right)$ and $K$ is a member of $T^{n}$, then in order that $P K P=K$ it is necessary and sufficient that $p_{i} K(i, j) p_{j}$ $=K(i, j)$ for $i, j=0, \cdots, n$.

Proof. If $y$ belongs to $A^{*}\left(S^{n}\right)$ then $p_{i} y_{i}=y_{i}(i=0, \cdots, n)$. It follows that the condition is necessary. To show that the condition is sufficient, it will be enough to show that if $y_{i}=D_{i}^{*} z_{i}(i=0, \cdots, n)$ for some $z$ in $S^{n}$ then $y$ is "approximately" in $A^{*}\left(S^{n}\right)$. Suppose, now, that $z$ is in $S^{n}$, that $y_{i}$ $=D_{i}^{*} z_{i}(i=0, \cdots, n)$, and that $c>0$. There is a member $x$ of $S^{n}$ such that $x_{n}=z_{n}$ and, for $i<n$, 


$$
N\left(D_{i}^{*} z_{i}-D_{i}^{*} x_{i}-D_{i}^{-1} \sum_{i+1}^{n} B(i, j) x_{j}\right)<c:
$$

hence $Q_{n}\left(y-A^{*} x, y-A^{*} x\right)<n c^{2}$, and the lemma is proved.

Lemma 11. Suppose $C$ is an infinite matrix with values in $T$ such that if $n$ is a positive integer then, for all $x$ in $S^{n}$,

$$
\sum_{i, j=0}^{n} Q\left(x_{i}, C(i, j) x_{j}\right) \geqq 0 .
$$

There is an infinite sequence $\Gamma$, each value of which is an infinite matrix with values in $T$, such that $\Gamma_{0}=C$ and, for each nonnegative integer $n, \Gamma_{n}(n, n) \gg 0$ and for each $A$ in $T$ such that $A A^{*}=\Gamma_{n}(n, n)$

$$
\Gamma_{n+1}(i, j)=\Gamma_{n}(i, j)-\left[A^{-1} \Gamma_{n}(n, i)\right]^{*}\left[A^{-1} \Gamma_{n}(n, j)\right]
$$

for $i, j=0,1,2, \cdots$; moreover, if $n$ is a positive integer then

(1) $\Gamma_{n}(i, j)=0$ if $i<n$ or $j<n$,

(2) if $\Gamma_{k}(k, k)=D_{k} D_{k}^{*}(k=0, \cdots, n)$ then

$$
C(i, j)=\sum_{k=0}^{n}\left[D_{k}^{-1} \Gamma_{k}(k, i)\right]^{*}\left[D_{\bar{k}^{-1}} \Gamma_{k}(k, j)\right] \text { if } i \leqq n \text { or } j \leqq n,
$$

(3) for each $z$ in $S, Q\left(z, \Gamma_{n}(n, n) z\right)$ is the greatest lower bound of

$$
\sum_{i, j=0}^{n} Q\left(x_{i}, C(i, j) x_{j}\right)
$$

for all $x$ in $S^{n}$ such that $x_{n}=z$.

REMARK. The nature of the sequence $\Gamma$ might be illustrated by a numerical example: for $C(i, j)=(i+j+1)^{-1}$, the formulas

$$
\Gamma_{n}(i, j)=\frac{\left(\begin{array}{l}
i \\
n
\end{array}\right)}{\left(\begin{array}{c}
i+n \\
n
\end{array}\right)} \frac{\left(\begin{array}{l}
j \\
n
\end{array}\right)}{\left(\begin{array}{c}
j+n \\
n
\end{array}\right)} \frac{1}{i+j+1}
$$

involving binomial coefficients, are easily established by induction.

Proof. This argument depends upon ideas developed in [10] in connection with kernel systems, and we sketch the relevant facts. Let $E$ denote the set of all nonnegative integers. The hypothesis on $C$ implies [10, Theorem 2.5] that there is only one complete inner product space $\left\{S^{\prime}, Q^{\prime}\right\}$, of functions from $E$ to $S$, in which $C$ is the kernel, i.e., in which it is true that for each $t$ in $E$ and $y$ in $S$ the function $C(\cdot, t) y$ belongs to $S^{\prime}$ and has the property that

$$
Q(f(t), y)=Q^{\prime}(f, C(\cdot, t) y) \quad \text { for all } f \text { in } S^{\prime} .
$$


The space $\left\{S^{\prime}, Q^{\prime}\right\}$ can be described as follows [10, Theorem 2.7]: the function $f$, from $E$ to $S$, belongs to $S^{\prime}$ only in case there exists a nonnegative number $b$ such that

$$
\left|\sum_{i} Q\left(f(i), x_{i}\right)\right|^{2} \leqq b \sum_{i, j} Q\left(x_{i}, C(i, j) x_{j}\right)
$$

for all finite sequences $x$ with values in $S$, in which case $Q^{\prime}(f, f)$ is the least such number $b$. Let $N^{\prime}$ be the norm corresponding to the inner product $Q^{\prime}$. Now $C(0,0) \gg 0$, since for each $x$ in $S$

$$
Q(x, C(0,0) x)=N^{\prime}(C(\cdot, 0) x)^{2} \geqq 0 .
$$

Let $D_{0}$ be a member of $T$ such that $D_{0} D_{0}^{*}=C(0,0)$. If $f$ is in $S^{\prime}$ and $y$ is in $S$ then

$$
|Q(f(0), y)|=\left|Q^{\prime}(f, C(\cdot, 0) y)\right| \leqq N^{\prime}(f) N\left(D_{0}^{*} y\right),
$$

so that, by Lemma $1, f(0)$ is in $D_{0}(S)$ and $N\left(D_{0}^{-1} f(0)\right) \leqq N^{\prime}(f)$; let $g$ be the function from $E$ to $S$ defined by

$$
g(i)=\left[D_{0}^{-1} C(0, i)\right]^{*}\left[D_{0}^{-1} f(0)\right] ;
$$

now, for each $z$ in $S$ and each finite sequence $x$ with values in $S$,

$$
Q\left(\sum_{i} C(0, i) x_{i}, z\right)=Q^{\prime}\left(\sum_{i} C(\cdot, i) x_{i}, C(\cdot, 0) z\right)
$$

so that

$$
\left|Q\left(\sum_{i} C(0, i) x_{i}, z\right)\right|^{2} \leqq \sum_{i, j} Q\left(x_{i}, C(i, j) x_{j}\right) N\left(D_{0}^{*} z\right)^{2},
$$

whence, by Lemma $1, \sum_{i} C(0, i) x_{i}$ is in $D_{0}(S)$ and

$$
N\left(D_{0}^{-1} \sum_{i} C(0, i) x_{i}\right)^{2} \leqq \sum_{i, j} Q\left(x_{i}, C(i, j) x_{j}\right) ;
$$

thus we see that, for each finite sequence $x$ with values in $S$,

$$
\begin{aligned}
\left|\sum_{i} Q\left(g(i), x_{i}\right)\right|^{2} & =\left|Q\left(D_{0}^{-1} f(0), D_{0}^{-1} \sum_{i} C(0, i) x_{i}\right)\right|^{2} \\
& \leqq N\left(D_{0}^{-1} f(0)\right)^{2} N\left(D_{0}^{-1} \sum_{i} C(0, i) x_{i}\right)^{2} \\
& \leqq N^{\prime}(f)^{2} \sum_{i, j} Q\left(x_{i}, C(i, j) x_{j}\right)
\end{aligned}
$$

hence $g$ belongs to $S^{\prime}$ and $N^{\prime}(g) \leqq N^{\prime}(f)$.

Let $P$ denote the subset of $S^{\prime} \times S^{\prime}$ to which $\{f, h\}$ belongs only in case 


$$
h(i)=f(i)-\left[D_{0}^{-1} C(0, i)\right]^{*}\left[D_{0}^{-1} f(0)\right] \quad \text { for all } i \text { in } E .
$$

By the preceding paragraph, we see that $P$ is a linear function from $S^{\prime}$ into $S^{\prime}$ which is continuous with respect to $N^{\prime}$; moreover, $P^{2}=P$ and $P f=f$ only in case $f(0)=0$. Let $K$ be the function from $E \times E$ to $T$ defined by

$$
K(i, j)=C(i, j)-\left[D_{0}^{-1} C(0, i)\right]^{*}\left[D_{0}^{-1} C(0, j)\right] .
$$

Now [10, Corollary 3.1c], $K$ is the matrix representation of $P$-in the sense that for all $f$ in $S^{\prime}$ and $i$ in $E$ and $x$ in $S$

$$
Q^{\prime}\left(P^{*} f, C(\cdot, i) x\right)=Q^{\prime}(f, K(\cdot, i) x)
$$

-and, since $K(i, j)^{*}=K(j, i)$ for all $i$ and $j$ in $E, P$ is Hermitian with respect to $Q^{\prime}$. Therefore $P$ is a projection with respect to $Q^{\prime}$, the projection of $S^{\prime}$ onto the subset of $S^{\prime}$ to which $f$ belongs only in case $f(0)=0$. Hence $K$ has the same nonnegative character as that postulated for $C$ and, by Remark 2 following Lemma 2, is independent of the choice of $D_{0}$ in $T$ such that $D_{0} D_{0}^{*}=C(0,0)$.

Now, what we did with $C$ at the integer 0 can be repeated with $K$ at the integer 1 . Thus we see that, with $\Gamma_{0}=C$ and $\Gamma_{1}=K$, the inductive definition of $\Gamma$ is justified. The properties (1) and (2) asserted for $\Gamma$ follow readily from our construction. Concerning (3) we see that for $z$ in $S$, and each $x$ in $S^{n}$ such that $x_{n}=z$,

$$
\begin{aligned}
\sum_{i, j=0}^{n} Q\left(x_{i}, C(i, j) x_{j}\right) & =\sum_{k=0}^{n} N\left(\sum_{i=k}^{n} D_{k}^{-1} \Gamma_{k}(k, i) x_{i}\right)^{2} \\
& =Q\left(z, \Gamma_{n}(n, n) z\right)+\sum_{k=0}^{n-1} N\left(D_{k}^{*} x_{k}+D_{k}^{-1} \sum_{k+1}^{n} \Gamma_{k}(k, i) x_{i}\right)^{2}
\end{aligned}
$$

hence, (3) may be established by the type of procedure employed in the latter part of our proof of Lemma 10, and this completes our proof of Lemma 11.

LEMma 12. With $C$ and $\Gamma$ as in Lemma 11, it is further true that if $C(i, j)=0$ for $i+j$ odd then, for each positive integer $n$, it is also true that $\Gamma_{n}(i, j)=0$ for $i+j$ odd.

Indication of proof. A simple induction argument can be made, to show that if $n$ is a nonnegative integer then

$$
\Gamma_{2 n+1}(i, j)= \begin{cases}0 & \text { if } i+j \text { is odd, } \\ \Gamma_{2 n}(i, j) & \text { if } i \text { or } j \text { is odd }\end{cases}
$$

and

$$
\Gamma_{2 n+2}(i, j)= \begin{cases}0 & \text { if } i+j \text { is odd } \\ \Gamma_{2 n+1}(i, j) & \text { if } i \text { or } j \text { is even. }\end{cases}
$$


We omit the details.

LEMma 13. If $M$ is an infinite matrix with values in $T$ such that $M(i, j)=0$ for $|i-j|>1$, then the following are true:

(1) If $n$ is a positive integer, $M^{n}(i, j)=0$ for $|i-j|>n$.

(2) If $n$ is a positive integer then the contraction $M_{n}$ of $M$ to the ordered pairs of integers in $[0, n]$ has the property that

$$
M_{n}^{k}(0,0)=M^{k}(0,0) \quad \text { for } k=1, \cdots, 2 n+1 .
$$

(3) If $M(i, i)=0(i=0,1, \cdots)$ then

$$
M^{2 k+1}(0,2 i)=M^{2 k+2}(0,2 i+1)=0 \quad(i, k=0,1,2, \cdots)
$$

REMARK. Using Lemma 13(3) and a simple induction argument, one is led to the following observation: if $M$ is an infinite matrix with values in $T$ such that $M(i, j)=0$ for $|i-j| \neq 1$ and $L$ is the infinite matrix defined by

$$
L(i, j)=M^{2}(2 i, 2 j) \quad(i, j=0,1,2, \cdots),
$$

then, for each positive integer $k, L^{k}(0, j)=M^{2 k}(0,2 j)(j=0,1, \cdots)$.

Indication of proof. Assertion (1) results from straightforward induction with the indicated matrix multiplication. Concerning (2), by supposing $n$ is a positive integer it is easy to establish-in order-the following facts:

(a) If $0<k<n$ and $M^{k}(0, j)=M_{n}^{k}(0, j)(j=0, \cdots, n)$ then $M^{k+1}(0, j)$ $=M_{n}^{k+1}(0, j)(j=0, \cdots, n)$.

(b) $M^{n+1}(0, j)=M_{n}^{n+1}(0, j)(j=0, \cdots, n)$.

(c) If $1 \leqq k \leqq n$ and $M^{n+k}(0, j)=M_{n}^{n+k}(0, j)$ for $0 \leqq j \leqq n-k+1$, then $M^{n+k+1}(0, j)=M_{n}^{n+k+1}(0, j)$ for $0 \leqq j \leqq n-k$.

Assertion (3) is obtained by straightforward induction on the matrix powers of $M$, starting from $M(0,2 i)=0(i=0,1, \cdots)$ and

$$
M^{2}(0,2 i+1)=M(0,2 i) M(2 i, 2 i+1)+M(0,2 i+2) M(2 i+2,2 i+1)=0 .
$$

We omit the details.

Definition. $S^{\infty}$ denotes the collection of all infinite sequences $x$ with values in $S$ such that the series $\sum_{i=0}^{\infty} N\left(x_{i}\right)^{2}$ converges, and $Q_{\infty}$ denotes the inner product for $S^{\infty}$ defined by

$$
Q_{\infty}(x, y)=\operatorname{Limit} \sum_{i=0}^{\infty} Q\left(x_{i}, y_{i}\right) ;
$$

we identify the class of linear functions from $S^{\infty}$ into $S^{\infty}$, which are continuous with respect to the norm $N_{\infty}$ corresponding to $Q_{\infty}$, with the class $T^{\infty}$ of infinite matrices $M$, with values in $T$, such that for some positive number $b$ and each $x$ and $y$ in $S^{\infty}$ 


$$
\left|\sum_{i, j=0}^{n} Q\left(x_{i}, M(i, j) y_{j}\right)\right|^{2} \leqq b \sum_{i=0}^{n} N\left(x_{i}\right)^{2} \sum_{j=0}^{n} N\left(y_{j}\right)^{2}
$$

for all positive integers $n$-the identification being made so that

$$
(M y)_{i}=\text { Limit } \sum_{j=0}^{\infty} M(i, j) y_{j} \quad(i=0,1, \cdots) .
$$

Lemma 14. Suppose $M$ is a member of $T^{\infty}$ such that $M(i, j)=0$ for $|i-j|$ $>1(i, j=0,1,2, \cdots), V(M)$ is the numerical range of $M$-the set of all complex numbers $w$ such that

$$
w=Q_{\infty}(M x, x) \quad \text { for some } x \text { in } S^{\infty} \text { with } Q_{\infty}(x, x)=1
$$

-and $U$ is the complement in the plane of the closure of $V(M)$, and $R$ is the resolvent of $M$ (so that, for all $z$ in $U, R[z]$ is the inverse of $z-M$ in $T^{\infty}$ ). For each $z$ in $U$

$R[z](0,0)=\operatorname{Limit}\left(z-B_{1}-A_{1}\left(z-B_{2}-A_{2}\left(z-B_{3}-\cdots\right)^{-1} C_{2}\right)^{-1} C_{1}\right)^{-1}$,

where $A_{p}=M(p-1, p), B_{p}=M(p-1, p-1), C_{p}=M(p, p-1)(p=1,2, \cdots)$, and convergence is uniform in $z$ over each closed bounded subset of $U$.

REMARK. For complex valued $M$ and $C_{p}=A_{p}(p=1,2, \cdots)$, this is precisely H. S. Wall's theorem $[20 ; 21$, p. 114] on convergence of bounded $J$ fractions.

Proof. Let $w$ be a point in $V(M), L$ be the infinite sequence with values in $T^{\infty}$ defined by:

$$
L_{n}(i, j)^{-}=\left\{\begin{array}{cl}
M(i, j) & \text { if } i \leqq n \text { and } j \leqq n, \\
w & \text { if } i=j>n, \\
0 & \text { if } i \neq j \text { and } i>n \text { or } j>n,
\end{array}\right.
$$

and $P_{n}$ be the resolvent of $L_{n}(n=0,1, \cdots)$. Since the set $V(M)$ is convex $[4 ; 17$, p. 131$]$, it is easy to see that $V\left(L_{n}\right)$ is a subset of $V(M)(n=0,1, \cdots)$; for each positive number $d$, each $z$ in $U$ at a distance not less than $d$ from $V(M)$, and each nonnegative integer $n$,

$$
\left\{\begin{aligned}
P_{n}[z]-R[z] & =P_{n}[z]\left\{L_{n}-M\right\} R[z], \text { and } \\
N_{\infty}\left(P_{n}[z] x\right) & \leqq N_{\infty}(x) / d \quad \text { for all } x \text { in } S^{\infty} .
\end{aligned}\right.
$$

Since $L$ has (strong) limit $M$ with respect to $N_{\infty}$ then, because of the indicated boundedness of $P, P$ has (strong) limit $R$ with respect to $N_{\infty}$ at each point of $U$; because of the analyticity of each value of $P$ and because of the aforesaid boundedness, this convergence is uniform in $z$ over each closed bounded subset of $U$.

For each $z$ in $U, P_{0}[z](0,0)=\left(z-B_{1}\right)^{-1}$. Also, because of the convexity of 
$V(M)$, one can use Theorem 11 and Lemma $\mathrm{A}$ of [9] to show that for each $z$ in $U$

$$
P_{1}[z](0,0)=\frac{1}{z-B_{1}-A_{1}} \cdot \frac{C_{1}}{z-B_{2}}=\left[z-B_{1}-A_{1}\left(z-B_{2}\right)^{-1} C_{1}\right]^{-1}
$$

and, for each integer $n$ greater than 1 ,

$$
\begin{aligned}
P_{n}[z](0,0)=\frac{1}{z-B_{1}-\cdot} & \\
\cdot & -A_{n} \cdot \frac{C_{n}}{z-B_{n+1}} .
\end{aligned}
$$

Hence Lemma 14 is established.

Lemma 15. Suppose $M$ is an infinite matrix with values in $T$ such that $M(i, j)=0$ for $|i-j|>1(i, j=0,1,2, \cdots)$, and for each positive integer $n$ and each $x$ in $S^{n}$

$$
\operatorname{Im} \sum_{i, j=0}^{n} Q\left(M(i, j) x_{j}, x_{i}\right) \leqq 0 .
$$

There exists a function $\Phi$ from the real line into $T^{\infty}$, nondecreasing with respect to $Q_{\infty}$, with Stieltjes transform $F$ and $\Phi(-\infty)=0$ and $\Phi(+\infty)=1$ in $T^{\infty}$, such that the following statement (1) is true; moreover, for each $\Phi$ such that (1) holds, (2) is also true:

(1) For $\operatorname{Im} z>0$ and $i, j=0,1,2, \cdots$,

$$
\sum_{k}\left\{z \cdot \delta_{i k}-M(i, k)\right\} F[z](k, j)=\sum_{k} F[z](i, k)\left\{z \cdot \delta_{k j}-M(k, j)\right\}=\delta_{i j} \text {. }
$$

(2) If $M(i, j)^{*}=M(j, i)(i, j=0,1,2, \cdots)$ then, for $n=1,2, \cdots$,

$$
M^{n}(i, j)=\int I^{n} d \Phi[I](i, j) \quad(i, j=0,1,2, \cdots) .
$$

Indication of proof. Part (1) is a direct application of Lemma 7 in the space $\left\{S^{\infty}, Q_{\infty}\right\}$, where we make the following identification: $S_{0}$ is the subset of $S^{\infty}$ to which $x$ belongs only in case there is a positive integer $n$ such that $x_{n+k}=0(k=0,1, \cdots)$, and for each $x$ in $S_{0}$ and each nonnegative integer $i$

$$
(A x)_{i}=\sum_{j} M(i, j) x_{j} \quad \text { and } \quad(B x)_{i}=\sum_{j} M(j, i)^{*} x_{j}
$$

Using (1) and Lemma 13(1), we see that for each positive integer $n$

$$
z^{n+1} F[z](i, j)-z^{n} \delta_{i j}-\sum_{k=1}^{n-1} z^{n-k} M^{k}(i, j)=M^{n}(i, j)+\sum_{k=0}^{i+n+1} M^{n+1}(i, k) F[z](k, j)
$$


for $\operatorname{Im} z>0$ and $i, j=0,1,2, \cdots$. Hence, if $m$ is a positive integer, and $B$ is the infinite sequence with values in $T^{m}$ such that $B_{0}=1$ in $T^{m}$ and $B_{k}$ is the contraction of $M^{k}$ to the ordered pairs of integers in $[0, m](k=1,2, \cdots)$, and $g(z)$ (for $\operatorname{Im} z>0$ ) is the contraction of $F[z]$ to the ordered pairs of integers in $[0, m]$, we can carry out the type of argument used in connection with Lemma $5(2)$ to establish the conclusion (2) for $i, j=0, \cdots, m$. This is enough to establish Lemma 15.

\section{Principal theorems.}

THEOREM 1. Suppose $\phi$ is a nondecreasing function from the real line into $T$ such that all the moments $\int I^{n} d \phi(n=0,1, \cdots)$ exist, $\phi(u)=\phi(u+)-\phi(-\infty)$ for all real $u, D_{0}$ is a member of $T$ such that $D_{0} D_{0}^{*}=\phi(+\infty)$, and $f$ is the Stieltjes transform of $\phi$. There exists a function $\Phi$ from the real line into $T^{\infty}$, nondecreasing with respect to $Q_{\infty}$ with $\Phi(u)=\Phi(u+)-\Phi(-\infty)$ for all real $u$ and $\Phi(+\infty)$ $=1$ in $T^{\infty}$, and an infinite matrix $M$, with values in $T$ and $M(i, j)=0$ for $|i-j|>1$ and $M(i, j)^{*}=M(j, i)(i, j=0,1,2, \cdots)$, such that

(1) if $F$ is the Stieltjes transform of $\Phi$ in $T^{\infty}$ then, for $\operatorname{Im} z>0$ and $i, j=0,1,2, \cdots, f(z)=D_{0} F[z](0,0) D_{0}^{*}$ and

$$
\sum_{k}\left\{z \cdot \delta_{i k}-M(i, k)\right\} F[z](k, j)=\sum_{k} F[z](i, k)\left\{z \cdot \delta_{k j}-M(k, j)\right\}=\delta_{i j},
$$

(2) $\phi(u)=D_{0} \Phi[u](0,0) D_{0}^{*}$ for all real $u$ and, for each positive integer $k$,

$$
M^{k}(i, j)=\int I^{k} d \Phi[I](i, j) \quad(i, j=0,1,2, \cdots) .
$$

REMARK. As will be apparent from the following argument, if $T_{0}$ is a subset of $T$ such that each $H \gg 0$ in $T$ has a factorization of the form $D D^{*}=H$ with $D$ in $T_{0}$ then the matrix $M$ can be so chosen that $M(i, i+1)$ is in $T_{0}(i=0,1, \cdots)$; for example, $T_{0}$ might be taken to be the class of all nonnegative Hermitian members of $T$ or, if $S$ is finite dimensional and $T$ is represented by a collection of complex matrices, $T_{0}$ might be taken as a corresponding collection of matrices with "only zeros above the main diagonal" and nonnegative real numbers on the main diagonal.

Indication of proof. With $\phi, D_{0}$, and $f$ as postulated, we apply Lemmas 5 and 6 , inductively to obtain infinite sequences $A$ and $B$, each with values in $T$, and infinite sequences $g$ and $\theta$ such that

(a) $A_{0}=D_{0}$ and $\theta_{0}$ is a nondecreasing function from the real line to $T$, with Stieltjes transform $g_{0}$ and $\theta_{0}(u)=\theta_{0}(u+)-\theta_{0}(-\infty)$ for all real $u$ and $\theta_{0}(+\infty)=1$, such that $f(z)=A_{0} g_{0}(z) A_{0}^{*}$ for $\operatorname{Im} z>0$, and

(b) for $p=1,2, \cdots, B_{p}=\int I d \theta_{p-1}, A_{p} A_{p}^{*}=\int I^{2} d \theta_{p-1}-B_{p}^{2}$, and $\theta_{p}$ is a nondecreasing function from the real line to $T$, with Stieltjes transform $g_{p}$ and $\theta_{p}(u)=\theta_{p}(u+)-\theta_{p}(-\infty)$ for all real $u$ and $\theta_{p}(+\infty)=1$, such that for $\operatorname{Im} z>0$

$$
z-B_{p}-A_{p} g_{p}(z) A_{p}^{*}=g_{p-1}(z)^{-1} \text {. }
$$


Let $M$ be the infinite matrix (with values in $T$ ) defined by $M(i, j)=0$ if $|i-j|>1, M(i+1, i)^{*}=M(i, i+1)=A_{i+1}, M(i, i)=B_{i+1}$, and $F$ be a function from the upper half of the complex plane into the set of infinite matrices with values in $T$ such that, for $\operatorname{Im} z>0, F[z](0,0)=g_{0}(z)$ and $(i=0,1, \cdots ; p=1,2, \cdots)$

$$
\begin{aligned}
F[z](p, p) & =g_{p}(z)+g_{p}(z) A_{p}^{*} F[z](p-1, p-1) A_{p} g_{p}(z), \\
F[z](i, i+p) & =F[z](i, i+p-1) A_{i+p} g_{i+p}(z), \\
F[z](i+p, i) & =g_{i+p}(z) A_{i+p}^{*} F[z](i+p-1, i) .
\end{aligned}
$$

Now, an easy computation (or translation into the notation of $[9$, Lemma A]) shows that, for $\operatorname{Im} z>0, F[z]$ is a reciprocal of the matrix $z \cdot \delta-M$ in the sense of (1) of this Theorem. Moreover, by our Representation Theorem [ 9 , p. 676], the fact that

$$
\operatorname{Im}\left[A_{p} g_{p}(z) A_{p}^{*}\right] \ll 0 \quad \text { for } \operatorname{Im} z>0(p=1,2, \cdots)
$$

implies that $F$ is the Stieltjes transform of a function $\Phi$ from the real line into $T^{\infty}$, nondecreasing with respect to $Q_{\infty}$, such that $\Phi(u)=\Phi(u+)-\Phi(-\infty)$ for all real $u$ and $\Phi(+\infty)=1$ in $T^{\infty}$. The proof is completed by application of Lemma 15, with the observation $[8$, p. 162] that $f$ determines $\phi$ uniquely.

Theовем 2. Suppose $C$ is an infinite sequence with values in $T$ and $D_{0}$ is a member of $T$ such that $D_{0} D_{0}^{*}=C_{0}$. If $M$ is an infinite matrix with values in $T$ such that $M(i, j)=0$ for $|i-j|>1$ and $M(i, j)^{*}=M(j, i)(i, j=0,1,2, \cdots)$ and $D_{0} M^{k}(0,0) D_{0}^{*}=C_{k}$ for each positive integer $k$, then

(1) $C$ is an Hermitian moment sequence, and

(2) if $n$ is a nonnegative integer and $\left[a_{n}, b_{n}\right]$ is a number interval such that

$$
a_{n} \sum_{i=0}^{n} Q\left(x_{i}, x_{i}\right) \leqq \sum_{i, j=0}^{n} Q\left(x_{i}, M(i, j) x_{j}\right) \leqq b_{n} \sum_{i=0}^{n} Q\left(x_{i}, x_{i}\right)
$$

for each sequence $\left\{x_{i}\right\}_{0}^{n}$ with values in $S$, then for each such $\left\{x_{i}\right\}_{0}^{n}$

$$
a_{n} \sum_{i, j=0}^{n} Q\left(x_{i}, C_{i+j} x_{j}\right) \leqq \sum_{i, j=0}^{n} Q\left(x_{i}, C_{i+j+1} x_{j}\right) \leqq b_{n} \sum_{i, j=0}^{n} Q\left(x_{i}, C_{i+j} x_{j}\right) .
$$

Proof. Concerning conclusion (1), we apply Lemma 15 to $M$ and obtain an appropriate function $\Phi$ from the real line into $T^{\infty}$; now, with $\phi=D_{0} \Phi[I](0,0) D_{0}^{*}$ it is easy to see that $\phi$ is a nondecreasing function from the real line into $T$ and that

$$
C_{n}=\int I^{n} d \phi \quad(n=0,1, \cdots)
$$

thus establishing (1). Concerning (2), it is clear that 
$a_{0} \ll M(0,0) \ll b_{0}$ implies that $a_{0} C_{0} \ll C_{1} \ll b_{0} C_{0}$.

Suppose, then, that $n$ is a positive integer and $\left[a_{n}, b_{n}\right]$ has the indicated property with respect to $M$. Now the contraction $M_{n}$, of $M$ to the ordered pairs of integers in $[0, n]$, is a member of $T^{n}$ and is Hermitian with respect to $Q_{n}$. Let $\Phi$ be the function from the real numbers to $T^{n}$ which is the spectral resolution of $M_{n}$ in the space $\left\{S^{n}, Q_{n}\right\}$, modified so as to have at $a_{n}$ the value 0 in $T^{n}$. Letting $\theta=\Phi[I](0,0)$, we see that for each positive integer $k$

$$
M_{n}^{k}(0,0)=\int_{a_{n}}^{b_{n}} I^{k} d \theta
$$

Now, by application of Lemma 13, we see that

$$
C_{k}=D_{0}\left\{\int_{a_{n}}^{b_{n}} I^{k} d \theta\right\} D_{0}^{*} \quad \text { for } k=0, \cdots, 2 n+1 .
$$

Hence, for each $x$ in $S^{n}$ we have

$$
\sum_{i, j=0}^{n} Q\left(x_{i}, C_{i+j+1} x_{j}\right)=\sum_{i, j=0}^{n} Q\left(D_{0}^{*} x_{i},\left\{\int_{a_{n}}^{b_{n}} I^{i+j+1} d \theta\right\} D_{0}^{*} x_{j}\right)
$$

which can be written as

$$
\int_{a_{n}}^{b_{n}} I Q\left(\left[\sum_{i=0}^{n} I^{i} D_{0}^{*} x_{i}\right],\{d \theta\}\left[\sum_{j=0}^{n} I^{j} D_{0}^{*} x_{j}\right]\right),
$$

from which the desired conclusion follows. Theorem 2 is proved.

COROLlARY 2.1. The characterization (II) in the Introduction is a necessary and sufficient condition for the infinite sequence $C$ with values in $T$ to be an Hermitian moment sequence.

Theorem 3. Suppose $C$ is an infinite sequence with values in $T$ and, for each nonnegative integer $n,\left[a_{n}, b_{n}\right]$ is a number interval such that if $\left\{x_{i}\right\}_{0}^{n}$ is a sequence with values in $S$ then

$$
a_{n} \sum_{i, j=0}^{n} Q\left(x_{i}, C_{i+j} x_{j}\right) \leqq \sum_{i, j=0}^{n} Q\left(x_{i}, C_{i+j+1} x_{j}\right) \leqq b_{n} \sum_{i, j=0}^{n} Q\left(x_{i}, C_{i+j} x_{j}\right) .
$$

There is an infinite sequence $\Gamma$, each value of which is an infinite matrix with values in $T$, such that if each of $i$ and $j$ is a nonnegative integer then $\Gamma_{0}(i, j)$ $=C_{i+j}$ and, for each nonnegative integer $n, \Gamma_{n}(n, n) \gg 0$ and for each $A$ in $T$ such that $A A^{*}=\Gamma_{n}(n, n)$

$$
\Gamma_{n+1}(i, j)=\Gamma_{n}(i, j)-\left\{A^{-1} \Gamma_{n}(n, i)\right\} *\left\{A^{-1} \Gamma_{n}(n, j)\right\} ;
$$

if each of $D$ and $P$ is an infinite sequence with values in $T$ such that $D_{n} D_{n}$ 
$=\Gamma_{n}(n, n)$ and $P_{n}$ is the projection of $S$ onto the closure of $D_{n}^{*}(S)(n=0,1, \cdots)$, then for each nonnegative integer $n$

$$
C_{i+j}=\sum_{k=0}^{n}\left\{D_{k}^{-1} \Gamma_{k}(k, i)\right\} *\left\{D_{k}^{-1} \Gamma_{k}(k, j)\right\} \quad \text { if } i \leqq n \text { or } j \leqq n,
$$

and there is only one infinite matrix $M$ with values in $T$ such that

$$
C_{i+j+1}=\sum_{p=0}^{i} \sum_{q=0}^{j}\left\{D_{p}^{-1} \Gamma_{p}(p, i)\right\}^{*} M(p, q)\left\{D_{q}^{-1} \Gamma_{q}(q, j)\right\}
$$

and $P_{i} M(i, j) P_{j}=M(i, j)(i, j=0,1,2, \cdots)$; moreover, $M$ has the properties:

(1) $M(i, j)=0$ for $|i-j|>1$ and $M(i, j)^{*}=M(j, i)(i, j=0,1,2, \cdots)$.

(2) If $k$ is a positive integer then $D_{0} M^{k}(0,0) D_{0}^{*}=C_{k}$.

(3) For $n=0,1, \cdots$ and each sequence $\left\{x_{i}\right\}_{0}^{n}$ with values in $S$

$$
a_{n} \sum_{i=0}^{n} Q\left(x_{i}, P_{i} x_{i}\right) \leqq \sum_{i, j=0}^{n} Q\left(x_{i}, M(i, j) x_{j}\right) \leqq b_{n} \sum_{i=0}^{n} Q\left(x_{i}, P_{i} x_{i}\right) .
$$

(4) If $i$ is a nonnegative integer then $D_{i+1}(S)$ is a subset of $D_{i}(S)$ and $M(i, i+1)=D_{i}^{-1} D_{i+1}, M(0,0)=D_{0}^{-1}\left\{D_{0}^{-1} C_{1}\right\}^{*}$, and (if $\left.i>0\right)$

$$
M(i, i)=D_{i}^{-1}\left\{\left[D_{i}^{-1} \Gamma_{i}(i, i+1)\right]^{*}-\left[D_{i-1}^{-1} \Gamma_{i-1}(i-1, i)\right]^{*}\left[D_{i-1}^{-1} D_{i}\right]\right\} .
$$

Remark 1. With $C$ and $D$ as in Theorem 3, one can use Lemmas 1 and 11 to establish the following: the condition that $D_{n}^{-1}$ be in $T$ (for $n=0,1, \cdots$ ) is equivalent to the condition that there exist a positive numerical sequence $d$ such that (for $n=0,1, \cdots$ )

$$
\sum_{i, j=0}^{n} Q\left(x_{i}, C_{i+j} x_{j}\right) \geqq d_{n} Q\left(x_{n}, x_{n}\right) \quad \text { for all } x \text { in } S^{n} ;
$$

moreover, in case $S$ is finite dimensional, this is equivalent to

$$
\sum_{i, j=0}^{n} Q\left(x_{i}, C_{i+j} x_{j}\right)>0 \quad \text { for } Q\left(x_{n}, x_{n}\right)>0 .
$$

REMARK 2. With $\Gamma$ and $M$ as in Theorem 3, a simple induction can be used to extend property (2) of $M$ to the following: for $n=0,1, \ldots$

$$
\Gamma_{n}(i, j)=D_{0}\left\{\sum_{k \geqq n} M^{i}(0, k) M^{i}(k, 0)\right\} D_{0}^{*} \quad(i, j=0,1,2, \cdots) .
$$

Proof. The existence of the sequence $\Gamma$ follows, by Lemma 11, from the properties postulated for $C$ - since, for each nonnegative integer $n, b_{n}-a_{n}>0$ and if $x$ is in $S^{n}$ then

$$
\left(b_{n}-a_{n}\right) \sum_{i, j=0}^{n} Q\left(x_{i}, C_{i+j} x_{j}\right) \geqq 0 .
$$


Supposing, then, the sequences $D$ and $P$ as indicated, it follows from Lemmas 3 and 10 that there is only one member $G_{0}$ of $T$ such that

$$
C_{1}=D_{0} G_{0} D_{0}^{*} \quad \text { and } \quad P_{0} G_{0} P_{0}=G_{0}
$$

and that, for each positive integer $n$, there is only one member $G_{n}$ of $T^{n}$ such that (for $i, j=0, \cdots, n) P_{i} G_{n}(i, j) P_{j}=G_{n}(i, j)$ and

$$
C_{i+j+1}=\sum_{p, q=0}^{n}\left\{D_{p}^{-1} \Gamma_{p}(p, i)\right\}^{*} G_{n}(p, q)\left\{D_{q}^{-1} \Gamma_{q}(q, j)\right\} ;
$$

moreover, by the Remark following Lemma 3, $a_{n}\left(\operatorname{diag} P_{i}\right) \ll G_{n} \ll b_{n}\left(\operatorname{diag} P_{i}\right)$ in the space $\left\{S^{n}, Q_{n}\right\}$ and-from the structure of $\Gamma$ - for $i, j=0, \cdots, n$

$\left(\begin{array}{l}* \\ *\end{array}\right) \quad \sum_{q=0}^{j} G_{n}(i, q)\left\{D_{q}^{-1} \Gamma_{q}(q, j)\right\}=D_{i}^{-1} \Gamma_{i}(i, j+1)$.

From this latter fact it follows, inductively, that

$$
\sum_{q=0}^{j} G_{n}^{k}(i, q)\left\{D_{q}^{-1} \Gamma_{q}(q, j)\right\}=D_{i}^{-1} \Gamma_{i}(i, j+k)
$$

for $0 \leqq i \leqq n$ and $0 \leqq j+k \leqq n+1$, so that

$$
C_{k}=D_{0} G_{n}^{k}(0,0) D_{0}^{*} \quad(k=1, \cdots, n+1) .
$$

To complete a proof of all of our assertions in Theorem 3 , it is sufficient to show that $G_{n}(i, j)=0$ for $|i-j|>1$ and $G_{n+1}(i, j)=G_{n}(i, j)$ for $i, j=0, \cdots, n$ - provided that on the way we establish the appropriate analogue of assertion (4). This we now accomplish, using the relationships $\left({ }_{*}^{*}\right)$ and $P_{i} G_{n}(i, j) P_{j}$ $=G_{n}(i, j)=G_{n}(j, i)^{*}$ (and, implicitly, Lemma 1 to see that $D_{j+1}(S)$ is a subset of $\left.D_{j}(S)\right)$. For $j=0$ and $0 \leqq i \leqq n$, we have $G_{n}(i, 0) D_{0}^{*}=D_{i}^{-1} \Gamma_{i}(i, 1)$ so that

$$
\begin{aligned}
G_{n}(0,0) D_{0}^{*} & =D_{0}^{-1} C_{1}, \\
G_{n}(1,0) D_{0}^{*} & =D_{1}^{*}, \text { implying } G_{n}(0,1)=D_{0}^{-1} D_{1} \quad \text { if } n \geqq 1, \text { and } \\
G_{n}(i, 0) & =0 \quad \text { provided } 1<i \leqq n .
\end{aligned}
$$

Now, inductively, for $j \geqq 1$ and $0 \leqq i \leqq n$, we have

$$
\sum_{q=0}^{j-1} G_{n}(i, q)\left\{D_{q}^{-1} \Gamma_{q}(q, j)\right\}+G_{n}(i, j) D_{j}^{*}=D_{i}^{-1} \Gamma_{i}(i, j+1)
$$

so that

$$
G_{n}(j, j-1)\left\{D_{j-1}^{-1} \Gamma_{j-1}(j-1, j)\right\}+G_{n}(j, j) D_{j}^{*}=D_{j}^{-1} \Gamma_{j}(j, j+1),
$$

$G_{n}(j+1, j) D_{j}^{*}=D_{j+1}^{*}$, implying $G_{n}(j, j+1)=D_{j}^{-1} D_{j+1}$ if $n \geqq j+1$, and $G_{n}(i, j)=0$ provided $j+1<i \leqq n$. 
Omitting the few remaining details, this concludes our proof of Theorem 3.

COROLlary 3.1. The characterization (III) in the Introduction is a necessary and sufficient condition for the infinite sequence $C$ with values in $T$ to be an Hermitian moment sequence.

COROLlaRY 3.2. If $R$ is a proper subset of the real line which is closed, nondegenerate, and connected (i.e., an interval or a ray), and $C$ is an infinite sequence with values in $T$, the following are equivalent:

(1) There is a nondecreasing function $\phi$ from the real line into $T$, which is constant on each component of the complement of $R$, such that

$$
C_{n}=\int I^{n} d \phi \quad(n=0,1, \cdots) .
$$

(2) There is a member $A_{0}$ of $T$ such that $A_{0} A_{0}^{*}=C_{0}$ and an infinite matrix $M$, with values in $T$ and numerical range lying in $R$, such that $M(i, j)=0$ if $|i-j|>1(i, j=0,1,2, \cdots)$ and $A_{0} M^{k}(0,0) A_{0}^{*}=C_{k}(k=1,2, \cdots)$.

(3) There is, for each nonnegative integer $n$, a number interval $\left[a_{n}, b_{n}\right]$ lying in $R$ such that for each sequence $\left\{x_{i}\right\}_{0}^{n}$ with values in $S$

$$
a_{n} \sum_{i, j=0}^{n} Q\left(x_{i}, C_{i+j} x_{j}\right) \leqq \sum_{i, j=0}^{n} Q\left(x_{i}, C_{i+j+1} x_{j}\right) \leqq b_{n} \sum_{i, j=0}^{n} Q\left(x_{i}, C_{i+j} x_{j}\right) .
$$

REMARK. In (2) the numerical range of $M$ (supposed lying in $R$ ) is understood to be the set to which $w$ belongs only in case there is a nonnegative integer $n$ and a sequence $\left\{x_{i}\right\}_{0}^{n}$ with values in $S$ such that

$$
w=\sum_{i, j=0}^{n} Q\left(M(i, j) x_{j}, x_{i}\right) \quad \text { and } \quad \sum_{i=0}^{n} Q\left(x_{i}, x_{i}\right)=1 ;
$$

in this situation and in case $R$ is not bounded, instead of using our Lemmas 7 and 15 to exhibit a $\Phi$ and so deduce (1), one uses the fact (see, for example, [15a, p. 330]) that an associated Hermitian transformation has a self-adjoint extension in the space $\left\{S^{\infty}, Q_{\infty}\right\}$ with a spectral resolution $\Phi$ which is constant on the complement of $R$.

The following theorem is an elaboration of Remark 1 which follows Theorem 3. (See KreĬn [7] and Wall [22], for finite-dimensional S.)

THEOREM 4. If $C$ is an infinite sequence with values in $T$ and there is a positive numerical sequence $d$ such that

$$
\sum_{i, j=0}^{n} Q\left(x_{i}, C_{i+j} x_{j}\right) \geqq d_{n} Q\left(x_{n}, x_{n}\right)
$$

for $n=0,1, \cdots$ and each sequence $\left\{x_{i}\right\}_{0}^{n}$ with values in $S$, then $C$ satisfies the suppositions of Theorem 3 and so is an Hermitian moment sequence. 
Indication of proof. Supposing the infinite sequences $C$ and $d$ as indicated, let $\Gamma$ be the infinite sequence obtained from $C$ as in Theorem 3 , as guaranteed by Lemma 11 . Now $E_{n}=\Gamma_{n}(n, n)^{1 / 2}(n=0,1, \cdots)$ is reversibly continuous from $S$ onto $S$; moreover (for $n=0,1, \cdots$ )

$$
C_{i+j}=\sum_{k=0}^{n}\left\{E_{k}^{-1} \Gamma_{k}(k, i)\right\} *\left\{E_{k}^{-1} \Gamma_{k}(k, j)\right\} \quad \text { for } i, j=0, \cdots, n .
$$

Hence (for $n=0,1, \cdots$ ) there is a member $H_{n}$ of $T^{n}$ such that

$$
H_{n}^{-1}(i, j)=C_{i+j} \quad \text { for } i, j=0, \cdots, n \text {. }
$$

Thus we see that there is a positive numerical sequence $e$ such that

$$
\sum_{i, j=0}^{n} Q\left(x_{i}, C_{i+j} x_{j}\right) \geqq e_{n} \sum_{i=0}^{n} Q\left(x_{i}, x_{i}\right)
$$

for $n=0,1, \cdots$ and all $x$ in $S^{n}$; on the other hand, there is a positive numerical sequence $f$ such that, for $n=0,1, \cdots$ and all $x$ in $S^{n}$,

$$
-f_{n} \sum_{i=0}^{n} Q\left(x_{i}, x_{i}\right) \leqq \sum_{i, j=0}^{n} Q\left(x_{i}, C_{i+j+1} x_{j}\right) \leqq f_{n} \sum_{i=0}^{n} Q\left(x_{i}, x_{i}\right) \text {. }
$$

Therefore, with $a_{n}=-f_{n} / e_{n}$ and $b_{n}=f_{n} / e_{n}(n=0,1, \cdots), C$ has the properties postulated in Theorem 3 .

THEOREM 5. If $C$ is an infinite sequence with values in $T$ such that $C_{0} \gg 0$ and $M$ is an infinite matrix with values in $T$ such that $M(i, j)=0$ for $|i-j|>1$ and $M(i, j)^{*}=M(j, i)(i, j=0,1,2, \cdots)$, then

(1) if $M$ generates $C$ as in Theorem 2 and $M(i, i)=0(i=0,1, \cdots)$ then $C_{\llcorner n+1}=0(n=0,1, \cdots)$, while, conversely,

(2) if $C$ generates $M$ as in Theorem 3 and $C_{2 n+1}=0(n=0,1, \cdots)$ then $M(i, i)=0(i=0,1, \cdots)$.

Indication of proof. Apply Lemmas 13(3) and 12, respectively.

REMARK. From our development thus far, it is easy to assemble facts which support the equivalence of the following statements (for any infinite sequence $C$ with values in $T$ ):

(1) $C$ is an Hermitian moment sequence and $C_{2 n+1}=0(n=0,1, \cdots)$.

(2) There is a nondecreasing function $\phi$ from the real line to $T$, which is odd in the sense that $\phi(-u)=-\phi(u)$ for all real $u$, such that

$$
C_{n}=\int I^{n} d \phi \quad(n=0,1, \cdots) .
$$

(3) There is a member $D_{0}$ of $T$ such that $D_{0} D_{0}^{*}=C_{0}$ and an infinite matrix 
$M$ with values in $T$ such that $M(i, j)=0$ for $|i-j| \neq 1$ and $M(i, j)^{*}$ $=M(j, i)(i, j=0,1,2, \cdots)$ and $D_{0} M^{k}(0,0) D_{0}^{*}=C_{k}(k=1,2, \cdots)$.

(4) There is a nondecreasing function $\theta$ from the real line to $T$, which is constant on the set of negative numbers, such that

$$
C_{2 n}=\int I^{n} d \theta \quad \text { and } \quad C_{2 n+1}=0 \quad(n=0,1, \cdots) .
$$

(5) $C_{2 n+1}=0(n=0,1, \cdots)$ and there is a member $D_{0}$ of $T$ such that $D_{0} D_{0}^{*}=C_{0}$ and an infinite matrix $L$ with values in $T$ such that $L(i, j)=0$ for $|i-j|>1(i, j=0,1,2, \cdots), D_{0} L^{k}(0,0) D_{0}^{*}=C_{2 k}(k=1,2, \cdots)$, and

$$
\sum_{i, j=0}^{n} Q\left(x_{i}, L(i, j) x_{j}\right) \geqq 0
$$

for $n=0,1, \cdots$ and each sequence $\left\{x_{i}\right\}_{0}^{n}$ with values in $S$.

The following pair of theorems supplies a direct connection between (3) and (5), as suggested by the Remark following Lemma 13. The theorems are related to a sufficient condition, due to Stieltjes (see, for example, [21, p. $121]$ ), for a real $J$-fraction to be the even part of a continued fraction of a certain other type.

THEOREM 6.1. If $M$ is an infinite matrix with values in $T$ such that $M(i, j)$ $=0$ for $|i-j| \neq 1$ and $M(i, j)^{*}=M(j, i)(i, j=0,1,2, \cdots)$, and $L$ is the infinite matrix defined by

$$
L(i, j)=M^{2}(2 i, 2 j) \quad(i, j=0,1,2, \cdots),
$$

then $L(i, j)=0$ for $|i-j|>1$ and $L(i, j)^{*}=L(j, i)(i, j=0,1,2, \cdots), L^{k}(0,0)$ $=M^{2 k}(0,0)(k=1,2, \cdots)$, and

$$
\sum_{i, j=0}^{n} Q\left(x_{i}, L(i, j) x_{j}\right) \geqq 0
$$

for $n=0,1, \cdots$ and each sequence $\left\{x_{i}\right\}_{0}^{n}$ with values in $S$.

Proof. Supposing $M$ to be as indicated, let $A$ be an infinite sequence such that $A_{p}=M(p-1, p)(p=1,2, \cdots)$. All but the last of the assertions about $L$ follow from Lemma 13 and the immediately subsequent Remark. To establish the last assertion, we consider infinite sequences $a, b$, and $g$ such that $g_{0}=0$ and (for $p=1,2, \cdots$ )

$$
\begin{aligned}
a_{p} & =L(p-1, p)=A_{2 p-1} A_{2 p}, \quad b_{1}=L(0,0)=A_{1} A_{1}^{*}, \\
b_{p+1} & =L(p, p)=A_{2 p}^{*} A_{2 p}+A_{2 p+1} A_{2 p+1}^{*}, \text { and } \\
g_{p} & =A_{2 p}^{*} A_{2 p} \ll b_{p+1} ;
\end{aligned}
$$

now, for each $x$ and $y$ in $S$ and each positive integer $p$,

$$
\left|Q\left(x, a_{p} y\right)\right|^{2} \leqq Q\left(x, A_{2 p-1} A_{2 p-1}^{*} x\right) Q\left(y, A_{2 p}^{*} A_{2 p} y\right)=Q\left(x,\left[b_{p}-g_{p-1}\right] x\right) Q\left(y, g_{p} y\right) .
$$


Hence the last assertion is true, by Theorems 5 and 7 of [9].

THEOREM 6.2. If $L$ is an infinite matrix with values in $T$ such that $L(i, j)=0$ for $|i-j|>1$ and $L(i, j)^{*}=L(j, i)(i, j=0,1,2, \cdots)$ and

$$
\sum_{i, j=0}^{n} Q\left(x_{i}, L(i, j) x_{j}\right) \geqq 0
$$

for $n=0,1, \cdots$ and each sequence $\left\{x_{i}\right\}_{0}^{n}$ with values in $S$, then there exists an infinite sequence $g$ and an infinite sequence $u$, each with values in $T$, such that for each nonnegative integer $n$

(i) $g_{0}=0$ and $0 \ll g_{n} \ll L(n, n)$,

(ii) $u_{n}^{*} u_{n}$ is the projection of $S$ onto the closure of $g_{n}(S)$, and

(iii) $u_{n+1} g_{n+1}^{1 / 2}=\left[L(n, n)-g_{n}\right]^{-1 / 2} L(n, n+1)$;

if, moreover, $M$ is the infinite matrix defined by

$$
\begin{aligned}
M(2 i, 2 i+1) & =\left[L(i, i)-g_{i}\right]^{1 / 2}, \quad M(2 i+1,2 i+2)=u_{i+1} g_{i+1}^{1 / 2}, \\
M(i, j) & =0 \text { for }|i-j| \neq 1, \text { and } M(i, j)^{*}=M(j, i),
\end{aligned}
$$

then $M^{2}(2 i, 2 j)=L(i, j)(i, j=0,1,2, \cdots)$.

Proof. Supposing $L$ to have the indicated properties, then, by Theorem 8 of [9], $L(0,0) \gg 0$ and there exists an infinite sequence $g$, with values in $T$, such that $g_{0}=0$ and (for $n=0,1, \cdots$ )

$$
\left\{\left[L(n, n)-g_{n}\right]^{-1 / 2} L(n, n+1)\right\}^{*}\left\{\left[L(n, n)-g_{n}\right]^{-1 / 2} L(n, n+1)\right\}=g_{n+1}
$$

and $g_{n+1} \ll L(n+1, n+1)$. Now, by Lemma 1 , for $n=0,1, \cdots$ we have

$$
g_{n+1}^{1 / 2}(S)=\left\{\left[L(n, n)-g_{n}\right]^{-1 / 2} L(n, n+1)\right\}^{*}(S),
$$

and, by Lemma 2 , the infinite sequence $u$, such that $u_{0}=0$ and

$$
u_{n+1}^{*}=g_{n+1}^{-1 / 2}\left\{\left[L(n, n)-g_{n}\right]^{-1 / 2} L(n, n+1)\right\}^{*} \quad(n=0,1, \cdots),
$$

has the property that $u_{n}^{*} u_{n}$ is the projection of $S$ onto the closure of $g_{n}^{1 / 2}(S)$ - so that $g_{n}^{1 / 2} u_{n}^{*} u_{n} g_{n}^{1 / 2}=g_{n}(n=0,1, \cdots)$. Hence, letting $M$ be defined as indicated, we have (for $i=0,1, \cdots$ )

$$
\begin{gathered}
M^{2}(2 i, 2 i)=g_{i}^{1 / 2} u_{i}^{*} u_{i} g_{i}^{1 / 2}+L(i, i)-g_{i}=L(i, i), \text { and } \\
M^{2}(2 i, 2 i+2)=M(2 i, 2 i+1) M(2 i+1,2 i+2)=L(i, i+1) .
\end{gathered}
$$

Since the closure of $g_{n}(S)$ is the closure of $g_{n}^{1 / 2}(S)(n=0,1, \cdots)$, the proof is complete.

No proof will be offered for the following theorem, since (1) and (2) are equivalent by Lemma 8 , while (1) and (3) and (4) are equivalent by Corollary 3.2. 
THEOREM 7. If $C$ is an infinite sequence with values in $T$ and $[a, b]$ is a number interval, the following are equivalent:

(1) There is a nondecreasing function $\phi$ from $[a, b]$ to $T$ such that

$$
C_{n}=\int_{a}^{b} I^{n} d \phi \quad(n=0,1, \cdots) .
$$

(2) If $E$ is the class of real polynomials restricted to $[a, b]$ and $L$ is the function from $E$ to $T$ defined by

$$
L\left(\sum_{p=0}^{m} r_{p} \cdot I^{p}\right)=\sum_{p=0}^{m} r_{p} \cdot C_{p}
$$

then $L(f) \gg 0$ for each nonnegative $f$ in $E$.

(3) For $n=0,1, \cdots$ and each sequence $\left\{x_{i}\right\}_{0}^{n}$ with values in $S$

$$
a \sum_{i, j=0}^{n} Q\left(x_{i}, C_{i+j} x_{j}\right) \leqq \sum_{i, j=0}^{n} Q\left(x_{i}, C_{i+j+1} x_{j}\right) \leqq b \sum_{i, j=0}^{n} Q\left(x_{i}, C_{i+j} x_{j}\right) .
$$

(4) There is a member $D_{0}$ of $T$ such that $D_{0} D_{0}^{*}=C_{0}$ and an infinite matrix $M$ with values in $T$ such that $M(i, j)=0$ for $|i-j|>1$ and $M(i, j)^{*}=M(j, i)$ $(i, j=0,1,2, \cdots), D_{0} M^{k}(0,0) D_{0}^{*}=C_{k}(k=1,2, \cdots)$, and

$$
a \sum_{i=0}^{n} Q\left(x_{i}, x_{i}\right) \leqq \sum_{i, j=0}^{n} Q\left(x_{i}, M(i, j) x_{j}\right) \leqq b \sum_{i=0}^{n} Q\left(x_{i}, x_{i}\right)
$$

for $n=0,1, \cdots$ and each sequence $\left\{x_{i}\right\}_{0}^{n}$ with values in $S$.

This theorem, supplemented by Lemma 14, has the following two immediate corollaries.

COROLLARY 7.1. If $\phi$ is a nondecreasing function from the number interval $[a, b]$ into $T$ then there exists an infinite sequence $A$ and an infinite sequence $B$, each with values in $T$, such that

(1) if $M$ is the infinite matrix (with values in $T$ ) defined by $M(i, i)=B_{i+1}, M(i+1, i)^{*}=M(i, i+1)=A_{i+1}, M(i, j)=0$ for $|i-j|>1$ then, for $n=0,1, \cdots$ and each sequence $\left\{x_{i}\right\}_{0}^{n}$ with values in $S$,

$$
a \sum_{i=0}^{n} Q\left(x_{i}, x_{i}\right) \leqq \sum_{i, j=0}^{n} Q\left(x_{i}, M(i, j) x_{j}\right) \leqq b \sum_{i=0}^{n} Q\left(x_{i}, x_{i}\right),
$$

(2) for complex $z$ in the region $\operatorname{Ext}[a, b]$

$$
\int_{a}^{b} \frac{1}{z-I} d \phi=\text { Limit } A_{0}\left(z-B_{1}-A_{1}\left(z-B_{2}-\cdots\right)^{-1} A_{1}^{*}\right)^{-1} A_{0}^{*},
$$

with uniform convergence over each closed bounded subset of $\operatorname{Ext}[a, b]$. 
COROLlaRy 7.2. If $[a, b]$ is a number interval then, for each ordered pair $\{A, B\}$ of infinite sequences, with values in $T$ such that statement (1) in the preceding Corollary is true, there is a nondecreasing function $\phi$ from $[a, b]$ into $T$ such that (2) holds.

THEOREM 8. If $C$ is an infinite sequence with values in $T$, the following are equivalent:

(1) There is a nondecreasing function $\phi$ from $[0,1]$ to $T$ such that

$$
C_{n}=\int_{0}^{1} I^{n} d \phi \quad(n=0,1, \cdots) .
$$

(2) The sequence $C$ is totally monotone in the sense that

$$
\Delta^{m} C_{n}=\sum_{i=0}^{m}(-1)^{i}\left(\begin{array}{c}
m \\
i
\end{array}\right) C_{i+n} \gg 0 \quad(m, n=0,1,2, \cdots) .
$$

(3) There is an infinite sequence $A$ with values in $T$ such that, if $M$ is the infinite matrix (with values in $T$ ) defined by

$$
M(i+1, i)^{*}=M(i, i+1)=A_{i+1}, M(i, j)=0 \quad \text { for }|i-j| \neq 1,
$$
then

(a) $A_{0} A_{0}^{*}=C_{0}$ and $A_{0} M^{2 k}(0,0) A_{0}^{*}=C_{k}(k=1,2, \cdots)$ and

(b) for $n=0,1, \cdots$ and each sequence $\left\{x_{i}\right\}_{0}^{n}$ with values in $S$

$$
-\sum_{i=0}^{n} Q\left(x_{i}, x_{i}\right) \leqq \sum_{i, j=0}^{n} Q\left(x_{i}, M(i, j) x_{j}\right) \leqq \sum_{i=0}^{n} Q\left(x_{i}, x_{i}\right) .
$$

REMARK. By Theorem 7 of [9] and Theorem 6.2 of the present paper, each of the following is an equivalent formulation of the foregoing condition (b) on the sequence $A$ :

$\left(b^{\prime}\right)$ There is an infinite sequence $g$ with values in $T$ such that if $p$ is a positive integer then $0 \ll g_{p-1} \ll 1$ and

$$
\left|Q\left(x, A_{p} y\right)\right|^{2} \leqq Q\left(x,\left[1-g_{p-1}\right] x\right) Q\left(y, g_{p} y\right) \quad \text { for all } x \text { and } y \text { in } S .
$$

$\left(\mathrm{b}^{\prime \prime}\right)$ There is an infinite sequence $m$ and an infinite sequence $u$, each with values in $T$, such that $m_{0}=0$ and (for each positive integer $p$ ) $0 \ll m_{p} \ll 1, u_{p}^{*} u_{p}$ is the projection of $S$ onto the closure of $m_{p}(S), A_{p}(S)$ is a subset of $\left[1-m_{p-1}\right]^{1 / 2}(S)$, and

$$
\left[1-m_{p-1}\right]^{-1 / 2} A_{p}=u_{p} m_{p}^{1 / 2} \text {. }
$$

Indication of proof. If (1) is true then so is (2), since

$$
\Delta^{m} C_{n}=\int_{0}^{1}[1-I]^{m} \cdot I^{n} d \phi \quad(m, n=0,1,2, \cdots) .
$$

If (2) is true and $E$ is the class of real polynomials restricted to $[0,1]$ and $L$ is the function from $E$ to $T$ defined as in Theorem $7(2)$ then, since 


$$
L\left([1-I]^{m} \cdot I^{n}\right)=\Delta^{m} C_{n} \quad(m, n=0,1,2, \cdots),
$$

we see by Lemma 9 that $L(f) \gg 0$ for each nonnegative $f$ in $E$ : hence, by Lemma $8,(1)$ is true. Now, (1) is clearly equivalent to:

(4) There is a nondecreasing odd function $\theta$ from $[-1,1]$ into $T$ such that

$$
C_{n}=\int_{-1}^{1} I^{2 n} d \theta \quad(n=0,1, \cdots) .
$$

This establishes the Theorem, since (3) and"(4) are easily seen to be equivalent by Theorems 3, 5, and 7 .

COROLLARY 8.1. If $\phi$ is a nondecreasing function from $[0,1]$ to $T$ then there is an infinite sequence $A$, with values in $T$ satisfying condition ( $\left.\mathrm{b}^{\prime \prime}\right)$ of the Remark following Theorem 8, such that for complex $z$ in the region Ext [-1, 1]

$$
\int_{0}^{1} \frac{1}{z^{2}-I} d \phi=\text { Limit } A_{0}\left(z-A_{1}\left(z-A_{2}(z-\cdots)^{-1} A_{2}^{*}\right)^{-1} A_{1}^{*}\right)^{-1} A_{0}^{*},
$$

with uniform convergence on each closed bounded subset of Ext $[-1,1]$; conversely, each such sequence $A$ determines a nondecreasing $\phi$ such that this continued fraction expansion holds.

COROLlaRy 8.2. If $C$ is a totally monotone sequence with values in $T$ then there is an infinite sequence $A$, with values in $T$ satisfying condition ( $\left.\mathrm{b}^{\prime \prime}\right)$ of the Remark following Theorem 8, such that for complex $z$ in the (open) unit disc

$$
\sum_{n=0}^{\infty} z^{n} C_{n}=\text { Limit } A_{0}\left(1-z A_{1}\left(1-z A_{2}(1-\cdots)^{-1} A_{2}^{*}\right)^{-1} A_{1}^{*}\right)^{-1} A_{0}^{*} \text {, }
$$

where (if $0<r<1$ ) convergence is uniform for $|z| \leqq r$; conversely, each such sequence $A$ determines a totally monotone sequence $C$ such that this expansion holds.

REMARK. This is the form of the theorem, mentioned in our Introduction, first discovered by Wall [19] for numerical sequences. It can be obtained directly from Corollary 8.1 (with Theorem 8 ) by an "equivalence transformation" on the continued fraction, or from Theorem 8 together with a variation of Lemma 14 applied to the matrix $z \cdot M$, followed by a replacement of $z^{2}$ by $z$.

4. Functions of bounded variation. In mimicry of the standard idea for complex valued functions, it is customary to say that a function $\gamma$ from $[0,1]$ to $T$ is of bounded variation with respect to the norm $\bar{N}$ provided there is a number $k$ such that

$$
\sum_{i} \bar{N}\left(\gamma\left(u_{i}\right)-\gamma\left(u_{i-1}\right)\right) \leqq k
$$

for all finite nondecreasing sequences $u$ with values in $[0,1]$; if $S$ is not finite 
dimensional, there are nondecreasing functions from $[0,1]$ to $T$ which are not of bounded variation with respect to $\bar{N}$ (spectral resolutions of members $H$ of $T$ such that $0 \ll H \ll 1)$. Let us observe that the function $\gamma$, from $[0,1]$ to the complex numbers, is of bounded variation only in case there is an ordered pair $\{\alpha, \beta\}$ of nondecreasing functions from $[0,1]$ to the numbers such that

$$
|\gamma(v)-\gamma(u)|^{2} \leqq[\alpha(v)-\alpha(u)][\beta(v)-\beta(u)]
$$

for each subinterval $[u, v]$ of $[0,1]$. Accordingly we introduce the following definition, which provides us with an extension of Theorem 8 and with an extension of the Boas-Pólya theorem [1], as promised in our Introduction.

DEFINITION. The class BVQ, of functions of bounded variation with respect to $Q$, is the set to which $\gamma$ belongs only in case $\gamma$ is a function from the real line to $T$ and there exists an ordered pair $\{\alpha, \beta\}$ of nondecreasing functions from the real line to $T$ such that each of $\int d \alpha$ and $\int d \beta$ exists and

$$
|Q(x,[\gamma(v)-\gamma(u)] y)|^{2} \leqq Q(x,[\alpha(v)-\alpha(u)] x) Q(y,[\beta(v)-\beta(u)] y)
$$

for each $x$ and $y$ in $S$ and each number interval $[u, v]$; in this case, the ordered pair $\{\alpha, \beta\}$ is said to be a dominant pair for $\gamma$.

REMARK 1. This idea allows immediate generalization (which will not concern us here) to a setting, for instance, in which each of $\alpha, \beta$, and $\gamma$ is replaced by a finitely additive set function with values in the space of linear mappings from $X^{*}$ to $X$-where $X$ is a linear topological space with adjoint space $X^{*}$.

REMARK 2. The nondecreasing function $\phi$ from the real line to $T$ belongs to BVQ only in case $\int d \phi$ exists, in which case $\{\phi, \phi\}$ is a dominant pair for $\phi$ (Schwarz's inequality).

REMARK 3. If $f$ is a continuous function from, for instance, $[0,1]$ to the complex numbers, and $\gamma$ is in BVQ with dominant pair $\{\alpha, \beta\}$, then the Stieltjes integral $\int_{0}^{1} f d \gamma$ exists as a limit with respect to the norm $\bar{N}$ of appropriate approximating sums - as can be deduced from the following symbolic computation:

$$
\begin{aligned}
\left|Q\left(x, \sum f_{i} \cdot[d \gamma]_{i} y\right)\right| & \leqq \sum\left|Q\left(x, f_{i} \cdot[d \gamma]_{i} y\right)\right| \\
& \leqq \sum Q\left(x,\left|f_{i}\right|[d \alpha]_{i} x\right)^{1 / 2} Q\left(y,\left|f_{i}\right|[d \beta]_{i} y\right)^{1 / 2} \\
& \leqq\left\{\sum\left|f_{i}\right| Q\left(x,[d \alpha]_{i} x\right)\right\}^{1 / 2}\left\{\sum\left|f_{j}\right| Q\left(y,[d \beta]_{j} y\right)\right\}^{1 / 2},
\end{aligned}
$$

whence $\bar{N}\left(\sum f_{i} \cdot[d \gamma]_{i}\right) \leqq\left\{\max \left|f_{i}\right|\right\} \bar{N}(\alpha(1)-\alpha(0)) \bar{N}(\beta(1)-\beta(0))$.

REMARK 4. With reference to Hellinger integrals, as developed in [10, especially Theorems $1.1,1.2$, and 1.7, and remarks on p. 259], we observewith the help of Lemma 1 of this paper-the following facts: if $\gamma$ is in BVQ with dominant pair $\{\alpha, \beta\}$ then the integral

$$
\int[d \gamma]^{*}[d \alpha]^{-1}[d \gamma]=\int\left\{[d \alpha]^{-1 / 2}[d \gamma]\right\}^{*}\left\{[d \alpha]^{-1 / 2}[d \gamma]\right\}
$$


exists and, for each number interval $[u, v]$,

$$
\int_{u}^{v}[d \gamma]^{*}[d \alpha]^{-1}[d \gamma] \ll \beta(v)-\beta(u)
$$

conversely, if $\gamma$ is a function from the real line to $T$, and $\phi$ is a nondecreasing function from the real line to $T$ such that the integral $\int[d \gamma]^{*}[d \phi]^{-1}[d \gamma]$ exists, and $\beta$ is a nondecreasing function such that

$$
\beta(0)=0 \text { and } \beta(v)-\beta(u)=\int_{u}^{v}[d \gamma]^{*}[d \phi]^{-1}[d \gamma] \quad \text { for } u<v,
$$

and $\int[d \gamma][d \beta]^{-1}[d \gamma]^{*}$ exists, then $\gamma$ is in BVQ with dominant pair $\{\alpha, \beta\}$ such that

$$
\alpha(0)=0 \text { and } \alpha(v)-\alpha(u)=\int_{u}^{v}[d \gamma][d \beta]^{-1}[d \gamma]^{*} \quad \text { for } u<v .
$$

THEOREM 9. If $c$ is an infinite sequence with values in $T$ then the following are equivalent:

(1) There is a function $\gamma$ in BVQ such that

$$
c_{n}=\int_{0}^{1} I^{n} d \gamma \quad(n=0,1, \cdots) .
$$

(2) There exist totally monotone sequences $a$ and $b$, each with values in $T$, such that for all $x$ and $y$ in $S$

$$
\left|Q\left(x,\left[\Delta^{m} c_{n}\right] y\right)\right|^{2} \leqq Q\left(x,\left[\Delta^{m} a_{n}\right] x\right) Q\left(y,\left[\Delta^{m} b_{n}\right] y\right) \quad(m, n=0,1,2, \cdots) .
$$

Indication of proof. If each of $A, B$, and $C$ is a member of $T$, with $A \gg 0$ and $B \gg 0$, then $[9$, Theorem 5$]$ in order that

$$
|Q(x, C y)|^{2} \leqq Q(x, A x) Q(y, B y) \quad \text { for all } x \text { and } y \text { in } S,
$$

it is necessary and sufficient that

$$
\left(\begin{array}{ll}
A & C \\
C^{*} & B
\end{array}\right) \gg 0 \quad \text { as a member of } T^{1}
$$

-the space of continuous linear transformations in the space $\left\{S^{1}, Q_{1}\right\}$.Hence, the function $\gamma$ from the real line to $T$ belongs to BVQ only in case there are nondecreasing $\alpha$ and $\beta$ from the real line to $T$ such that

$$
\phi=\left(\begin{array}{ll}
\alpha & \gamma \\
\gamma^{*} & \beta
\end{array}\right)
$$

is a nondecreasing member of $\mathrm{BVQ}_{1}$. Thus we see that Theorem 9 is a direct application of Theorem 8 in the space $\left\{S^{1}, Q_{1}\right\}$. 
THEOREM 10. If $c$ is an infinite sequence with values in $T$ and $k$ is a number then there is a member $\gamma$ of $\mathrm{BVQ}$, with dominant pair $\{\alpha, \beta\}$, such that all the integrals $\int I^{n} d \alpha$ and $\int I^{n} d \beta(n=0,1, \cdots)$ exist,

$$
c_{n}=\int I^{n} d \gamma \quad(n=0,1, \cdots)
$$

and $\gamma$ is constant on the set of all numbers less than $k$.

Indication of proof. The idea, as in our proof of Theorem 9, is to make this an application of our other theorems in the space $\left\{S^{1}, Q_{1}\right\}$. More specifically, supposing $c$ to be an infinite sequence with values in $T$ and $k$ to be a positive number, we show the existence of infinite sequences $a$ and $b$ with values in $T$ such that the sequence $C$,

$$
C_{n}=\left(\begin{array}{cc}
a_{n} & c_{n} \\
c_{n}^{*} & b_{n}
\end{array}\right) \quad(n=0,1, \cdots),
$$

with values in $T^{1}$ has the property that

$$
\begin{gathered}
\sum_{i, j=0}^{n} Q_{1}\left(x_{i}, C_{i+j} x_{j}\right) \geqq\left(1+\frac{1}{n+1}\right) \sum_{i=0}^{n} Q_{1}\left(x_{i}, x_{i}\right) \text { and } \\
\sum_{i, j=0}^{n} Q_{1}\left(x_{i}, C_{i+j+1} x_{j}\right) \geqq\left(k+\frac{1}{n+1}\right) \sum_{i, j=0}^{n} Q_{1}\left(x_{i}, C_{i+j} x_{j}\right)
\end{gathered}
$$

for $n=0,1, \ldots$ and each sequence $\left\{x_{i}\right\}_{0}^{n}$ with values in $S^{1}$. Theorem 4 and Corollary 3.2, applied in the space $\left\{S^{1}, Q_{1}\right\}$, then supply a function $\phi$ from the real line to $T^{1}$, nondecreasing with respect to $Q_{1}$ and constant on the set of all numbers less than $k$, such that

$$
C_{n}=\int I^{n} d \phi \quad(n=0,1, \cdots) ;
$$

the identification of $\alpha, \beta$, and $\gamma$, with values in $T$ such that

$$
\phi=\left(\begin{array}{ll}
\alpha & \gamma \\
\gamma^{*} & \beta
\end{array}\right)
$$

then provides the asserted result. We now indicate how to obtain the conditions ( $\ddagger$ ).

First, we note that if $n$ is a nonnegative integer and $r$ is a positive number then there exists a member $a_{n}$ and a member $b_{n}$ of $T$ such that $C_{n} \gg r$ with respect to $Q_{1}$ : namely $a_{n}=b_{n} \gg \bar{N}\left(c_{n}\right)+r$. Hence, there exist members $a_{0}$ and $b_{0}$ of $T$ such that $C_{0} \gg 2$ with respect to $Q_{1}$, and then members $a_{1}$ and $b_{1}$ of $T$ such that $C_{1} \gg(k+1) C_{0}$ with respect to $Q_{1}$. 
Next, we note that if $n$ is a nonnegative integer and $\left\{A_{i}\right\}_{0}^{n+1}$ is a sequence with values in $T^{1}$ and $A_{n+1}$ is Hermitian with respect to $Q_{1}$ and $p$ is a positive number then there exists a member $B$ of $T^{1}$ such that

$$
p \sum_{i=0}^{n} Q_{1}\left(x_{i}, x_{i}\right)+2 \operatorname{Re} \sum_{i=0}^{n} Q_{1}\left(x_{n+1}, A_{i} x_{i}\right)+Q_{1}\left(x_{n+1},\left[B-A_{n+1}\right] x_{n+1}\right) \geqq 0
$$

for all sequences $\left\{x_{i}\right\}_{0}^{n+1}$ with values in $S^{1}$ : namely, $B$ in $T^{1}$ such that

$$
B \gg A_{n+1}+\frac{1}{p} \sum_{i=0}^{n} \bar{N}_{1}\left(A_{i}\right)^{2} \quad \text { with respect to } Q_{1},
$$

since the requirement is equivalent to the condition that

$$
\left|\sum_{i=0}^{n} Q_{1}\left(x_{n+1}, A_{i} x_{i}\right)\right|^{2} \leqq p Q_{1}\left(x_{n+1},\left[B-A_{n+1}\right] x_{n+1}\right) \sum_{i=0}^{n} Q_{1}\left(x_{i}, x_{i}\right)
$$

for all such $x$, and since Schwarz's inequality gives the estimate

$$
\left|\sum_{i=0}^{n} Q_{1}\left(x_{n+1}, A_{i} x_{i}\right)\right|^{2} \leqq Q_{1}\left(x_{n+1}, x_{n+1}\right) \sum_{i=0}^{n} \bar{N}_{1}\left(A_{i}\right)^{2} \sum_{j=0}^{n} Q_{1}\left(x_{j}, x_{j}\right) \text {. }
$$

Hence, supposing $n$ such that ( $\ddagger)$ holds for all $\left\{x_{i}\right\}_{0}^{n}$ with values in $S^{1}$, there exist $a_{2 n+2}$ and $b_{2 n+2}$ in $T$ such that

$$
\begin{aligned}
\left(\frac{1}{n+1}-\frac{1}{n+2}\right) \sum_{i=0}^{n} Q_{1}\left(x_{i}, x_{i}\right) & +2 \operatorname{Re} \sum_{j=0}^{n} Q_{1}\left(x_{n+1}, C_{n+1+j} x_{j}\right) \\
& +Q_{1}\left(x_{n+1},\left[C_{2 n+2}-\left(1+\frac{1}{n+2}\right)\right] x_{n+1}\right) \geqq 0
\end{aligned}
$$

for all $\left\{x_{i}\right\}_{0}^{n+1}$ with values in $S^{1}$, and then there exist $a_{2 n+3}$ and $b_{2 n+3}$ in $T$ such that

$$
\begin{aligned}
& \left(\frac{1}{n+1}-\frac{1}{n+2}\right) \sum_{i=0}^{n} Q_{1}\left(x_{i}, x_{i}\right) \\
& +2 \operatorname{Re} \sum_{j=0}^{n} Q_{1}\left(x_{n+1},\left[C_{n+2+j}-\left(k+\frac{1}{n+2}\right) C_{n+1+j}\right] x_{j}\right) \\
& \quad+Q_{1}\left(x_{n+1},\left[C_{2 n+3}-\left(k+\frac{1}{n+2}\right) C_{2 n+2}\right] x_{n+1}\right) \geqq 0
\end{aligned}
$$

for all such $\left\{x_{i}\right\}_{0}^{n+1}$ : it follows readily that ( $\left.\ddagger\right)$ holds with $n$ replaced by $n+1$. Therefore, an induction argument will yield the infinite sequence $C$, and this, as we have seen, is enough.

Supplementary comment. Attention is called to the paper by B. Sz.-Nagy ( $A$ moment problem for self-adjoint operators, Acta Math. Acad. Sci. Hungar. 
3 (1952), 285-293) for the prior equivalence of statements (1) and (2) in both Theorems 7 and 8 of the present paper, and to the paper by Chandler Davis ( $A$ device for studying Hausdorff moments, Trans. Amer. Math. Soc. 87 (1958), 144-158) for the prior equivalence of statements (1) and (4) in Theorem 7. Both papers deal with moments on an interval. It should also be noted that our Lemma 5 provides an extension of results obtained in the first of those two papers concerning the inequality $C_{1}^{2} \ll C_{2}$; this inequality seems first to have been studied by R. V. Kadison ( $A$ generalized Schwarz inequality and algebraic invariants for operator algebras, Ann. of Math. 56 (1952), 494-503); see also Remark 3 following Lemma 5 and concerning our earlier result $[8$, Theorem 3.4 , p. 167$]$. Finally, attention is called to the expository article by C. L. Dolph (Recent developments in some non-self-adjoint problems of mathematical physics, Bull. Amer. Math. Soc. 67 (1961), 1-69) for some ramifications of the theory of "dissipative operators" which we have studied as half-bounded matrices in [8] and in Lemma 7 of the present paper.

\section{BIBLIOGRAPHY}

1. R. P. Boas, The Stieltjes moment problem for functions of bounded variation, Bull. Amer. Math. Soc. 45 (1939), 399-404.

2. R. R. Christian, On order-preserving integration, Trans. Amer. Math. Soc. 86 (1957), 463-488.

3. H. Hamburger, Über eine Erweiterung des Stieltjesschen Momentenproblems. I, Math. Ann. 81 (1920), 235-319.

4. F. Hausdorff, Der Wertvorrat einer Bilinearform, Math. Z. 3 (1919), 314-316. 248.

5. - Über das Momentenproblem für ein endliches Interval, Math. Z. 16 (1923), 220-

6. A. Jakimovski, Some remarks on the moment problem of Hausdorff, J. London Math. Soc. 33 (1958), 1-13.

7. M. KreYn, Infinite J-matrices and a matrix-moment problem, Dokl. Akad. Nauk (N.S.) 69 (1949), 125-128.

8. J. S. Mac Nerney, Half-bounded matrices, J. Indian Math. Soc. 16 (1952), 151-176.

9. - Investigation concerning positive definite continued fractions, Duke Math. J. 26 (1959), 663-677.

10. - Hellinger integrals in inner product spaces, J. Elisha Mitchell Sci. Soc. 76 (1960), 252-273.

11. E. J. McShane, Order-preserving maps and integration processes, Ann. of Math. Studies No. 31, Princeton, N. J., 1953. 974-977.

12. F. Riesz, Sur les operations fonctionnelles linéaire, C. R. Acad. Sci. Paris 149 (1909),

13. - Les systèmes d'équations linéaires d une infinité d'inconnues, Gauthier-Villars, Paris, 1913.

14. - Zur Theorie des Hilbertschen Raumes, Acta Sci. Math. Szeged 7 (1934), 34-38.

15. F. Riesz and B. Sz.-Nagy, Lę̧ons d'analyse fonctionnelle, Akadémiai Kiad6, Budapest, 1952.

15a. - Functional analysis, Ungar, New York, 1955. (translation of second French edition of [15])

16. T. J. Stieltjes, Recherches sur les fractions continues, Ann. Fac. Sci. Univ. Toulouse 8 (1894), 1-122; 9 (1895), 1-47 (Oeuvres complètes, Vol. II, Groningen, 1918). 
17. M. H. Stone, Linear transformations in Hilbert space, Amer. Math. Soc. Colloq. Publ. 15, New York, 1932.

18. J. von Neumann, Functional operators. II, Annals of Mathematics Studies No. 22, Princeton, N. J., 1950.

19. H. S. Wall, Continued fractions and totally monotone sequences, Trans. Amer. Math. Soc. 48 (1940), 165-184.

20. —, Bounded J-fractions, Bull. Amer. Math. Soc. 52 (1946), 686-693.

21. - Analytic theory of continued fractions, Van Nostrand, New York, 1948.

22. —-, J-matrices of interior order $k$, Abstract 137t, Bull. Amer. Math. Soc. 57 (1951), 134.

23. J. H. Wells, Concerning the Hausdorff inclusion problem, Duke Math. J. 26 (1959), $629-645$.

University of North Carolina, Chapel Hill, North Carolina 